\title{
3. SOME CHARACTERISTICS OF INTERSTELLAR \\ GAS IN THE GALAXY
}

Introductory Report

(Tuesday, September 9, 1969)

HAROLD F. WEAVER

Radio Astronomy Laboratory, University of California, Berkeley, Calif., U.S.A.

\section{Task of the Review}

The task assigned for this review is to discuss structural features of the Galaxy as they may involve or relate to gas dynamics. Since a topic of such great breadth would permit discussion of essentially any aspect of the Galaxy, we shall narrow the view and direct attention primarily towards the larger-scale features of the system. In particular, effort will be made to place emphasis on those areas of the subject in which problems of interpretation exist and in which new theoretical models are needed.

\section{The Galaxy Among Others}

Examination of photographs of nearby galaxies similar to our own (see, for example, The Hubble Atlas of Galaxies, Sandage, 1961) reveals a number of general characteristics of such systems that should guide investigations of the large-scale properties of our own Galaxy.

(i) A galaxy normally exhibits a grand design, a two-armed (in some instances multi-armed) spiral winding outward from a spheroidal nucleus.

(ii) A spiral arm in this grand design is not a continuous structure; it appears to be composed of segments. On the largest scale, arms of some galaxies appear to be made of a series of almost straight-line sections; universally, on a smaller scale, the arms appear to be mottled and irregular, composed of clumps. Arms frequently are split and bifurcated.

(iii) In the majority of galaxies the arms are not more than $2 \pi$ or $3 \pi$ in angular length.

(iv) Not every feature in a galaxy fits into the grand design. There are many nonconforming structures, very often in the form of spurs (also called 'branches' or 'twigs' by some investigators) or interarm features. Spurs normally appear to originate on the outside of an arm and show a larger pitch angle (a factor of two is not unusual) than is exhibited by an arm in the grand design.

(v) Dark material is preferentially concentrated, often in a clumpy manner, at the inside edges of spiral arms. The outside edges of the arms are often less well-defined than the inner edges. The outside edges appear composed of material that is drawn out, coarsely 'brushed', from the inner edges. The 'brushed-out' clumps of material show larger pitch angles than the arm as a whole. In the inner parts of a galaxy the spiral arms may be delineated primarily by dark material. 
(vi) Arm and interarm regions appear to differ in character. Specifically, an arm does not appear to be just a region of slightly greater-than-average density.

(vii) Spiral arms and other features in a galaxy are generally narrow. Such generally compact delineation of the spiral arms must indicate that conditions suitable for stellar birth (at least for very massive stars) exist over very limited regions of the Galaxy - those same regions in which the massive stars are seen. During their mainsequence lifetimes most massive blue stars move less than $100 \mathrm{pc}$ (much less than the thickness of a spiral arm) from their places of birth, and even 'runaway' stars move only a kpc.

Clearly there must be much physics in progress in the arms of a galaxy. There the interstellar material is most plentiful. In the arms condensation takes place, stars are formed; we see the arms outlined by young stars, which are the principal spiral tracers. The interstellar medium may be expected to be quite different in character inside and outside the arms. To investigate such differences and to examine the gas structures that form the arms, we must turn to the Galaxy.

\section{The Grand Design in the Galaxy}

There are now two basic methods of establishing the nature of the grand design in our Galaxy.

(a) From optical observations we outline the arms by means of spiral tracers young stars, Hil regions, and the like - for which photometric distances can be derived.

(b) In the radio range we observe neutral hydrogen (which, by empirical evidence, is concentrated in the arms) and employ the galactic rotation curve to infer distances to the gas concentration from their observed Doppler velocities. (As a variant, in place of observations of $\mathrm{HI}$, one may observe radio recombination lines which originate in Hil regions.)

Neither of these methods is without shortcomings. Because of interstellar obscuration we can observe spiral tracers in the optical range over a very limited region of the Galaxy, within a distance of about $3 \mathrm{kpc}$ from the Sun, hence no truly large-scale pattern of arms can be delineated by optical techniques. The $1420 \mathrm{MHz}$ line of hydrogen can be observed throughout the entire extent of the Galaxy, but the gas in the system is not without radial motions of an apparently regional systematic character. Such non-circular motions are directly reflected in erroneous kinematic distances, hence the gas-derived picture of spiral structure will be regionally distorted by unknown amounts. In spite of this difficulty in distance determination, we must use $\mathrm{HI}$ as our probe if we are to determine the overall galactic spiral pattern. We have, in this case, no alternative to a kinematic distance scale.

If the pattern of spiral arms in the Galaxy is reasonably regular, we can determine one characteristic of the system, the pitch of the spiral arms, without recourse to any velocity measurement. From that pitch angle, we can provide an overall smooth representation of the Galaxy. 
In order to find the pitch angle we have only to determine the longitudes at which the line of sight is tangent to the next inner (Sagittarius) arm. We observe the longitudes of tangency to be $50^{\circ} .5$ and $284^{\circ} .0$; the derived pitch angle is $12^{\circ} .5$ (Weaver, 1970). We take the distance, $R_{0}$, from the galactic center to the Sun to be the standard value, $10 \mathrm{kpc}$, and employ the longitudes of tangency to find the constants of the equiangular spiral $R=2.00+2.20\left(\theta+{ }_{\pi}^{0}\right)$ by which we represent the galactic spiral. Here $\theta$

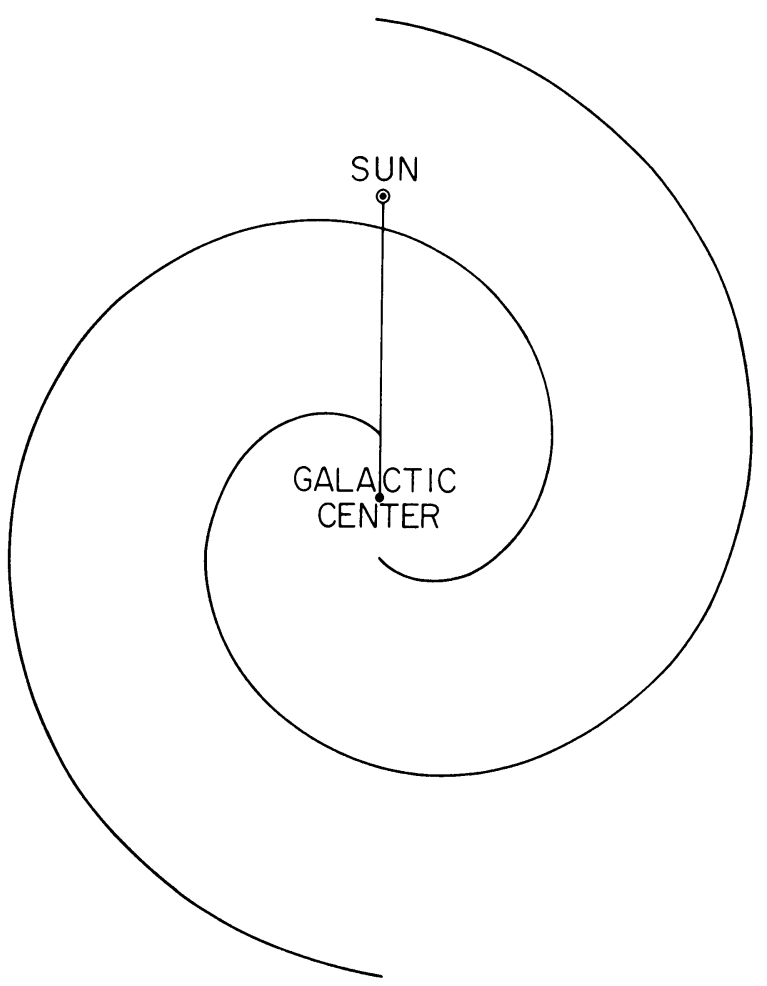

Fig. 1. Smooth, two-arm spiral with pitch angle $12^{\circ} .5$ representing the overall picture of our Galaxy.

represents galactocentric longitude. The smooth spiral galaxy thus derived is shown in Figure 1.

The real Galaxy is far more complex than the idealized system shown in Figure 1. We employ the extensive observations of $\mathrm{HI}_{\mathrm{I}}$ now available and utilize the rotation curve established by Schmidt (1965) to derive the galactic model shown in Figure 2a (Weaver, 1970). Shown in Figure 2b for comparison is the observed distribution of young stars and HII regions (Schmidt-Kaler, 1964).

In Figure 2 we note that the left and right sides of the spiral are quite different; more detail is shown on the right than on the left. This is observational only; it is not real. At present, hydrogen observations from the northern hemisphere are far more extensive and detailed than those from the southern hemisphere. The left-hand side 


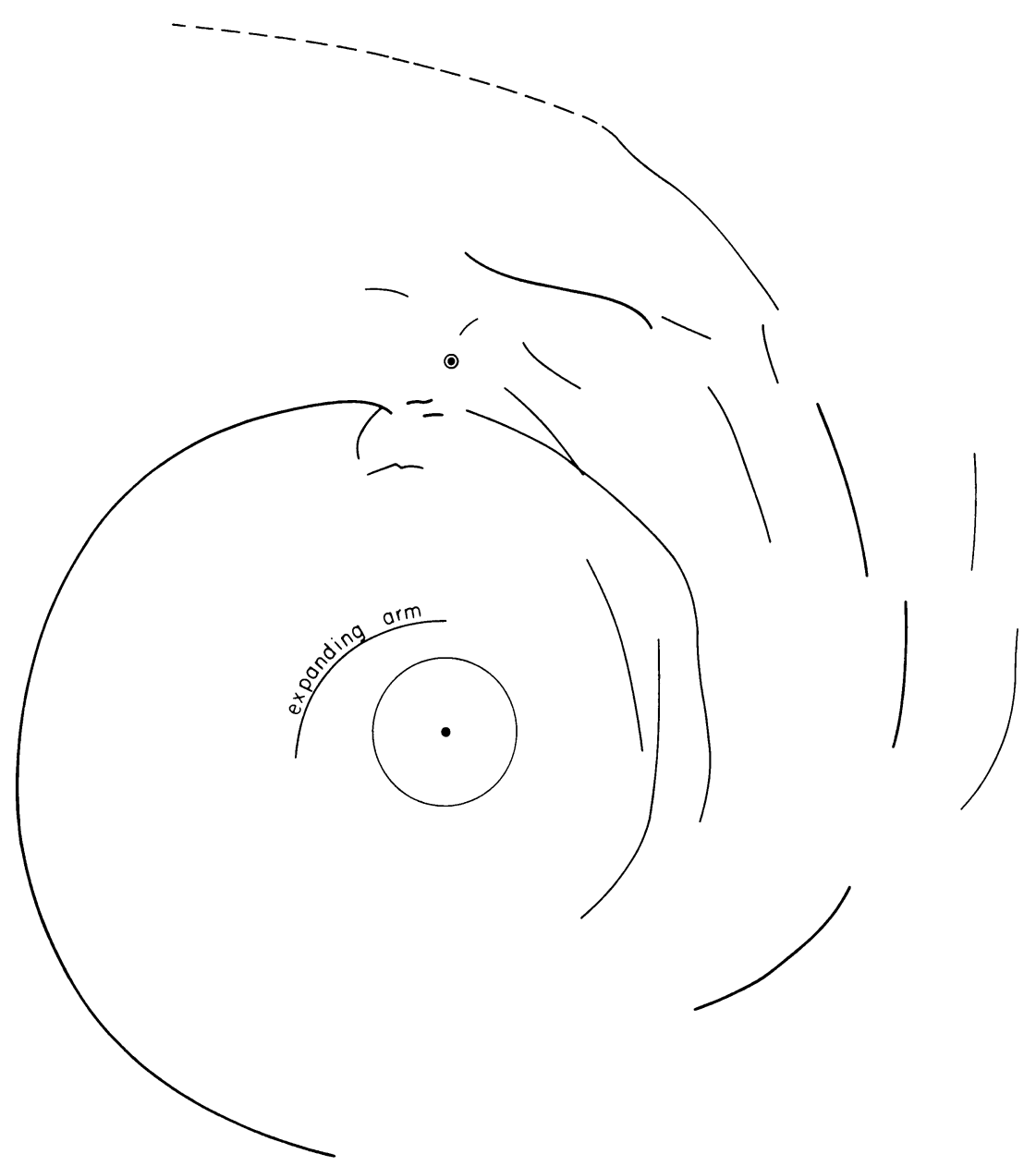

Fig. 2a. Diagram representing the structure of the Galaxy as derived from Hi observations. The left-hand side of the diagram is schematic. See the text for explanation of the difference between the two sides of the picture, which is not a real effect in the Galaxy.

of the figure is derived from southern-hemisphere observations limited in coverage. There is in reality much more detail present on the left side of the figure than is shown in the diagram, but that detail cannot now be delineated with the certainty desired because of the lack of a sufficiently close net of data points. Details were therefore omitted from the left side of the picture. Details on the right side can be drawn in with some assurance; the left side is a very rough sketch only.

In Figure 2 the arms are shown broken into bits and pieces. This characteristic may, in part, arise from regional non-circular motions of the type mentioned earlier. Clearly, however, there are many spurs and interarm links. The gas is concentrated in large complexes spirally distributed, but not all belonging to a smooth two-arm spiral.

There has long been a disagreement in arm pitch angle derived from stars and from 


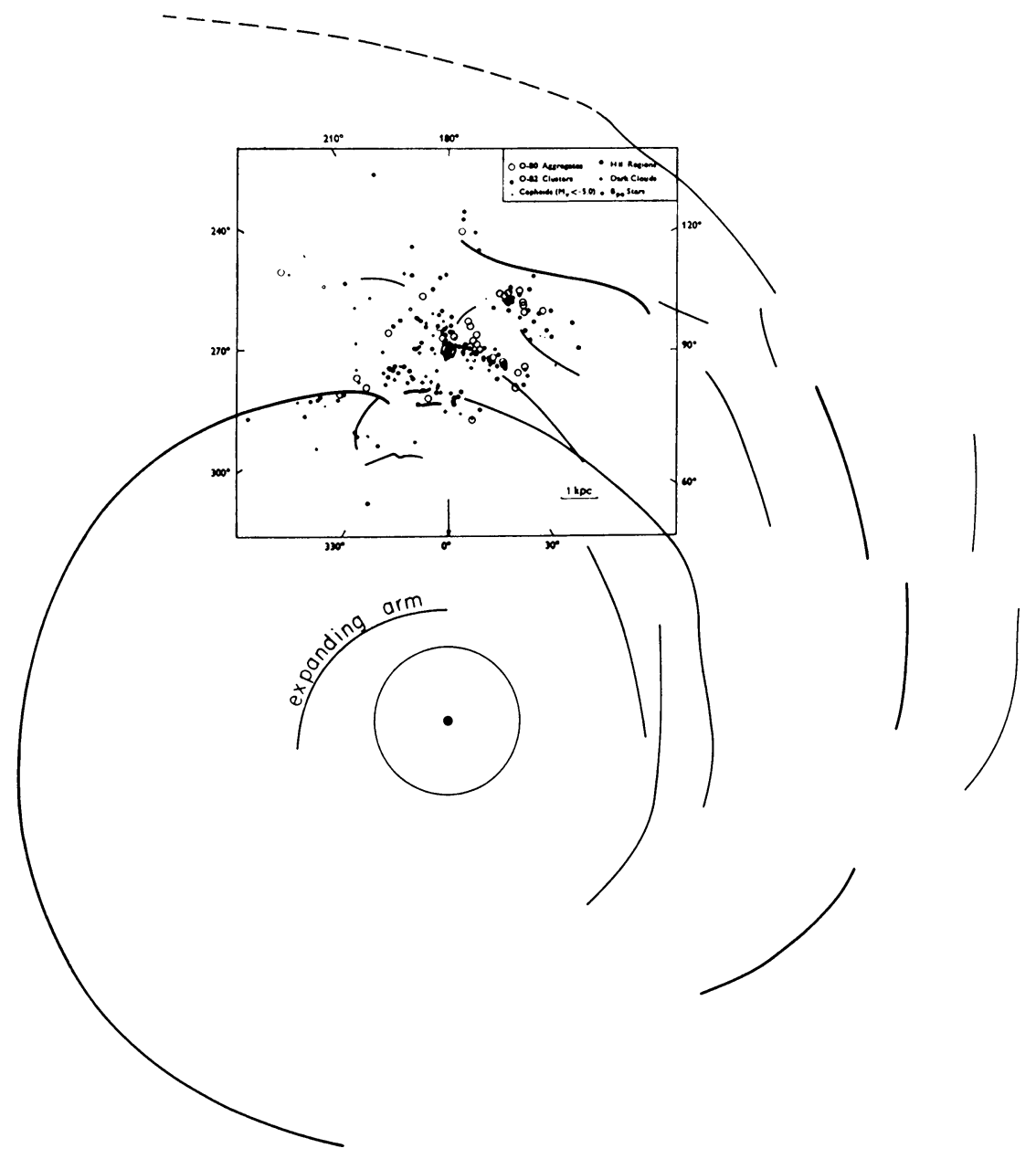

Fig. 2b. Picture identical with Figure 2a except that the spiral arms as outlined by stars is shown superimposed on the $\mathrm{HI}$ arms. See text for more complete explanation.

gas. The extensive observations now available make it clear that the Sun is not located in a major spiral arm, but in a spur or offshoot of the Sagittarius arm. If the pitch of the spur is derived from stellar observations and from radio observations, there is no disagreement. In the past, optical observations of the local (spur) structure have been compared with galactic-scale hydrogen observations. In such a comparison, disagreement is inevitable. A more complete discussion of this situation has been given by Weaver (1970).

Not given in Figure 2 is any indication of the cross-sectional thickness of the arm structures or the well-known warp of the galactic plane. Kerr (1969) treats the latter phenomenon. 


\section{Some Characteristics of the Gas in the System}

\section{A. THE NATURE OF AN ARM}

For the present we largely ignore the non-gaseous components of the Galaxy and use the term spiral arm to refer to a large-scale elongated gas structure of spiral form which fits into the grand design. Spurs and interarm features are numerous; presumably, these are structures similar to the arms in physical properties.

In the definition we use here we describe an arm in space in accord with the usual photographs we are accustomed to see. Operationally, we use a different coordinate system in studying spiral structures. We observe these gas structures in position on the sky (angular coordinates) with velocity replacing radial distance. This results in many troublesome ambiguities. Observations provide intensity or antenna temperature, $T_{A}$, of the radiation at each radial velocity, $v_{r}$, over some bandwidth, $\Delta v_{r}$, arising
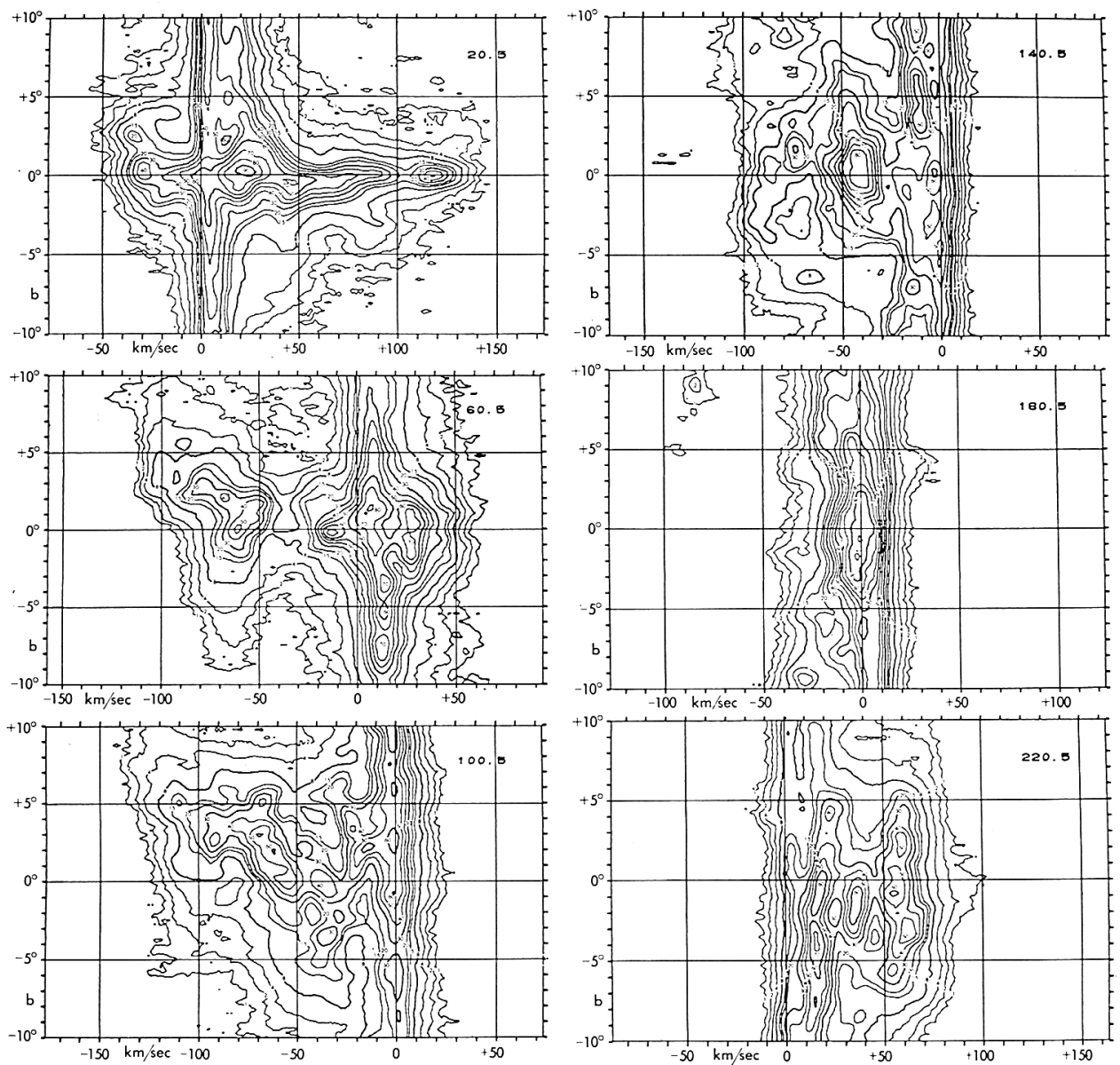

Fig. 3. Examples of contour maps $T_{A}\left(v_{r}, b \mid l\right)$ for a variety of $l$-values. 
from a point on the sky at longitude, $l$, and latitude, $b$. We display the data in the form of contour maps of antenna temperature as a function of radial velocity and one angle, keeping the second angle fixed. Alternatively, we may display the data as contour maps of antenna temperature as a function of two angles for a fixed velocity centered in bandwidth $\Delta v_{r}$. This latter display is a picture of gas intensity seen on the sky in a specific velocity range.

In Figure 3 we show, for several $l$-values, contour maps in the form $T_{A}$ as a function of $v_{r}$ and $b$ for fixed $l$. We designate such a map symbolically as the function $T_{A}\left(v_{r}, b \mid l\right)$. Such maps show clearly velocity cross cuts of spiral arms and spurs at a given $l$. From these particular maps the general nature of the gaseous arm structures is well seen; arm structures are separate entities (at least in velocity) within which there are subconcentrations of gas. From a series of such maps closely spaced in $l$, it is clear that the major structure we would term an arm persists over a long range in $l$, while any individual subconcentration within the arm persists over a very limited range in $l$ and $b$. The latter phenomenon is readily demonstrated by means of a $T_{A}\left(l, b \mid v_{r}\right)$ map, Figure 4, which shows the $l, b$ extent of the individual members of a group of subconcentrations.

Contour maps of these two kinds suggest several basic, gas-dynamically related questions that we now consider:

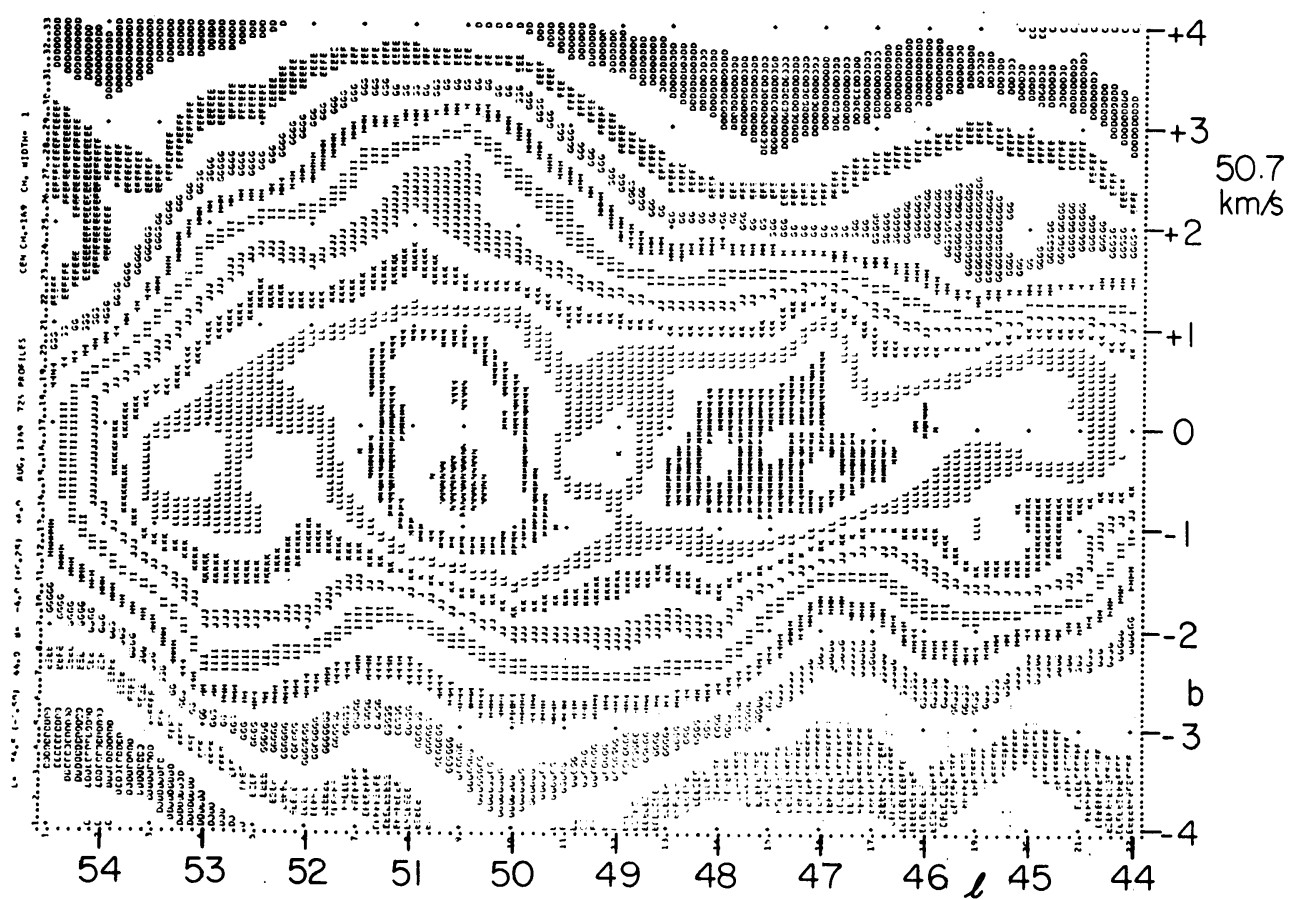

Fig. 4. $T_{A}\left(l, b \mid v_{r}\right)$ map for longitude range $44^{\circ}$ to $54^{\circ} .5$ illustrating clumpiness within a section of a spiral arm which shows continuity in longitude. 
(1) What is the arm/interarm contrast in number density of $\mathrm{HI}$ atoms?

(2) What are the statistical properties - linear scales, number densities, masses of the subconcentrations within the arm and other large-scale structures in the Galaxy?

(3) What are the velocity characteristics of the gas and the subunits of the gas forming an arm?

\section{B. ARM/INTERARM CONTRAST}

We can determine the arm/interarm contrast directly by measuring absorption by the HI gas in the arm and interarm regions along the line of sight to a strong radio source.

A concentration of cool $\mathrm{HI}$ gas in the line of sight will attenuate the radiation of the radio source lying beyond it. (For simplicity in this discussion we assume the source and the Hi cloud to be centered one over the other and of the same angular size and shape. We thus eliminate geometrical factors not necessary to the physical argument.) From the measured attenuation we compute the effective optical depth, $\tau_{\text {eff }}\left(v_{0}\right)$, of the Hi concentration at $v_{0}$, the frequency of maximum absorption. The optical depth $\tau_{\text {eff }}\left(v_{0}\right)$ is related to the physical parameters of the Hi gas by the equation

$$
\tau_{\text {eff }}\left(v_{0}\right)=q L\left\langle\frac{n_{\mathrm{H}}}{T_{\mathrm{ex}}}\right\rangle .
$$

Here $q$ is a numerical factor composed of atomic and numerical constants, $L$ represents the path length in the absorbing hydrogen, $n_{H}$ is the number density of the hydrogen, and $T_{\mathrm{ex}}$ is the excitation temperature, which describes the relative populations of two energy levels involved in the transition. For $\mathrm{HI}_{\mathrm{I}} T_{\mathrm{ex}}$ is equal to $T_{\mathrm{kin}}$. We shall take $T_{\mathrm{ex}}$ to be uniform throughout the HI concentration producing the absorption. Under this circumstance $\left\langle n_{\mathrm{H}} / T_{\mathrm{ex}}\right\rangle=\left\langle n_{\mathrm{H}}\right\rangle / T_{\mathrm{ex}}$.

The value of $T_{\mathrm{ex}}$ (as well as $\tau_{\mathrm{eff}}\left(v_{0}\right)$ ) can be determined from the absorption meaurements. Specifically,

$$
T_{\mathrm{ex}}=\left[T_{A}\left(v_{0}\right) / \Delta T_{A}\left(v_{0}\right)\right] T_{B, s}\left(v_{0}\right),
$$

where $T_{A}\left(v_{0}\right)$ represents the antenna temperature one would observe for the $\mathrm{HI}_{\mathrm{I}}$ if there were no absorption, $\Delta T_{A}\left(v_{0}\right)$ the depth of the absorption, and $T_{B, s}\left(v_{0}\right)$ the brightness temperature of the source, all at frequency $v_{0}$. From the derived values of $\tau_{\text {eff }}\left(v_{0}\right)$ and $T_{\text {ex }}$, one can determine $\left\langle n_{\mathrm{H}}\right\rangle$ provided an estimate of $L$ is available. If one makes observations of the absorption arising from the gas in arm and interarm regions along the line of sight, one can then determine the arm-interarm contrast, $\left\langle n_{\mathrm{H}}\right\rangle_{\mathrm{arm}} /$ $\left\langle n_{\mathrm{H}}\right\rangle_{\text {interarm }}$, which is what we require.

Alternatively, if pressure equilibrium exists between the arm and the interarm gas, as seems likely, one can estimate the arm-interarm contrast from the relation

$$
\left\langle n_{\mathrm{H}}\right\rangle_{\mathrm{arm}} /\left\langle n_{\mathrm{H}}\right\rangle_{\text {interarm }}=T_{\text {ex, interarm }} / T_{\text {ex, arm }} \text {. }
$$

We shall use both methods of estimating the contrast.

Figure 5 shows a contour map, $T_{A}\left(v_{r}, b \mid l\right)$ for the longitude of Cas A observed at Hat Creek (Weaver and Williams, 1970). We note immediately the strong absorp- 


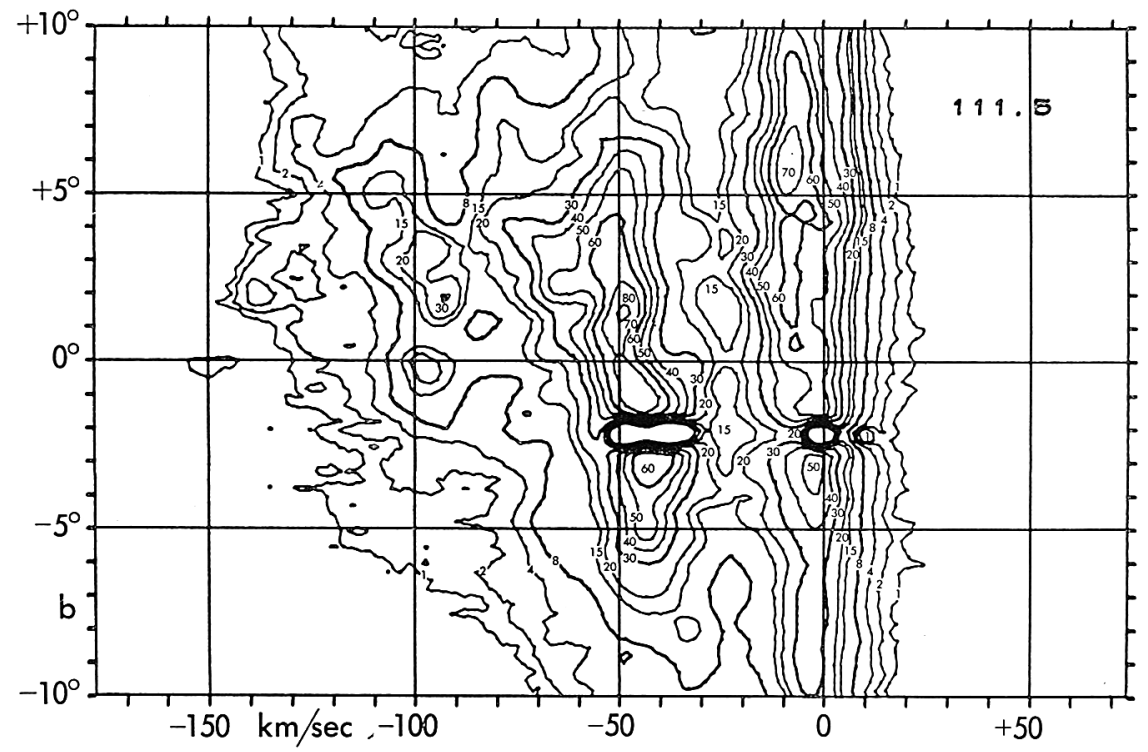

Fig. 5. Contour map showing $\mathrm{H} \mathrm{I}$ absorption resulting from the source Cas A.

tion in the arms, the weak absorption in the interarm region. Williams (1969) has analyzed the observations in the vicinity of Cas A and has found the $T_{\text {ex }}, \tau_{\text {eff }}\left(v_{0}\right)$ values along the line of sight to Cas A. He finds (see Figure 6) that in the arm $T_{\mathrm{ex}}$ is low, the $T_{\text {ex }}$ values derived from the negative velocity lines being $70 \mathrm{~K}$ and $85 \mathrm{~K}$. The zero-velocity line shows a temperature of $93 \mathrm{~K}$, while the $+9 \mathrm{~km} \mathrm{sec}^{-1}$ line corresponds to a temperature of $160 \mathrm{~K}$. By comparing widths of $\mathrm{H}$ and $\mathrm{OH}$ lines from the same source, Barrett et al. (1964) found for the components of the zero-velocity line (the line shows as a close-spaced doublet on $\mathrm{OH}$ ) kinetic temperatures of $90 \mathrm{~K}$ and $120 \mathrm{~K}$. Some lack of confidence has been expressed for temperatures derived from $\mathrm{H} / \mathrm{OH}$ line widths because of maser action frequently shown by the $\mathrm{OH}$ molecule. It is not likely that such trouble has sensibly affected the $1667 \mathrm{MHz}$ line involved in this comparison, though the satellite lines show peculiar intensities. The temperatures derived from absorption measurements and from $\mathrm{H} / \mathrm{OH}$ line width comparison are in excellent agreement. In the interarm region Williams finds that $T_{\mathrm{ex}}$ is high, $\approx 1000$ $3000 \mathrm{~K}$.

With the data derived by Williams we find for the arm/interarm regions crossed by the line of sight to Cas A

$$
\begin{aligned}
& \left\langle n_{\mathrm{H}}\right\rangle_{a} /\left\langle n_{\mathrm{H}}\right\rangle_{i a} \approx T_{i a} / T_{a} \approx 1500 / 90 \approx 17 \quad \text { (by Equation (3)) } \\
& \left\langle n_{\mathrm{H}}\right\rangle_{a} /\left\langle n_{\mathrm{H}}\right\rangle_{i a} \approx\left(\frac{\tau_{a}}{\tau_{i a}}\right)\left(\frac{L_{i a}}{L_{a}}\right)\left(\frac{T_{\mathrm{ex}, a}}{T_{\mathrm{ex}, i a}}\right) \approx \frac{0.6}{0.01} \times \frac{5}{1} \times \frac{80}{1500} \approx 16
\end{aligned}
$$

The arm-interarm contrast is in the range 15 to 20 .

(by Equations $(1,2)$ ). 
From similar observational data by Williams (1969) for the Tau A absorption, we estimate that the arm/interarm contrast along the line of sight to Tau $\mathrm{A}$ is $>20$.

Thus the conclusion from these two representative regions is that the arm-interarm contrast is generally high, $\approx 20$, and possibly even higher. However, this conclusion is open to the criticism that the values derived represent extreme upper limits since (a) the arms are represented by the lowest temperature objects within them and (b) the interarm regions may not have been adequately separated from influences of arm/ interarm interface regions; the temperature taken for the interarm region may be too low. Higher temperatures are recorded in the interarm regions; we employed an average that may not be representative. What is wanted for the determination of contrast, of course, is $\left\langle n_{\mathrm{H}}\right\rangle$ over a representative volume of the arm which includes concentrations and ambient gas, and a similarly representative volume of an interarm region. Adequately accurate values of these quantities are not now available; observers must make a strong effort to obtain such values. Very preliminary values estimated by methods of differing reliabilities suggest values for arm/interarm contrast which, though relatively high, are possibly half the value quoted. In no instance is there suggested a contrast ratio as low as, say, 3 .

\section{REMARKS ON THE OBSERVED TEMPERATURES OF THE GAS IN THE ARM AND INTERARM REGIONS}

Though we have some knowledge of the temperatures of $\mathrm{HI}$ concentrations in the arms, much remains to be learned. Observational knowledge is almost completely lacking about the temperature of (a) the gas in the arms in which the concentrations appear to be embedded, and (b) the interarm gas, which appears to be more generally free of concentrations than is the gas in the arms.

Under the gross simplification that within an arm or arm-like structure $n_{\mathrm{H}}$ and $T_{\mathrm{ex}}$ were space invariant, van de Hulst et al. (1954) and later Schmidt (1957) employed observed maximum antenna temperatures in directions little influenced by galactic rotation (Cygnus, the galactic center, and the galactic anti-center) to determine $T_{\mathbf{e x}}$. They estimated path lengths, $L$, within the relevant gas in each of the three named directions; Schmidt derived a universal $T_{\mathrm{ex}}$ of $125 \mathrm{~K}$.

However, at approximately the same time, Heeschen (1955) investigated hydrogen absorption in an extended cloud in the general direction of the galactic center and found $T_{\mathrm{ex}} \approx 40 \mathrm{~K}$. Davies $(1956,1958)$ derived similar temperatures for a cloud in Auriga. Later, Riegel and Jennings (1969) found an upper limit of $42 \mathrm{~K}$. and a more probable temperature of $20 \mathrm{~K}$ for an extension of the same object investigated by Heeschen. Clearly, objects of low temperature exist; the excitation temperature is not universal. From Figure 6 it is clear that regions of quite different temperatures exist within an arm or arm-like structure.

Shuter and Verschuur (1964), from a study of absorption lines, determined that H I concentrations in the so-called 'Orion Arm' (the local large-scale spur that contains the Sun) had temperatures ranging from $25 \mathrm{~K}$ to $120 \mathrm{~K}$, with a harmonic mean temperature of $56 \mathrm{~K}$, a value quite different from the then frequently quoted and generally 

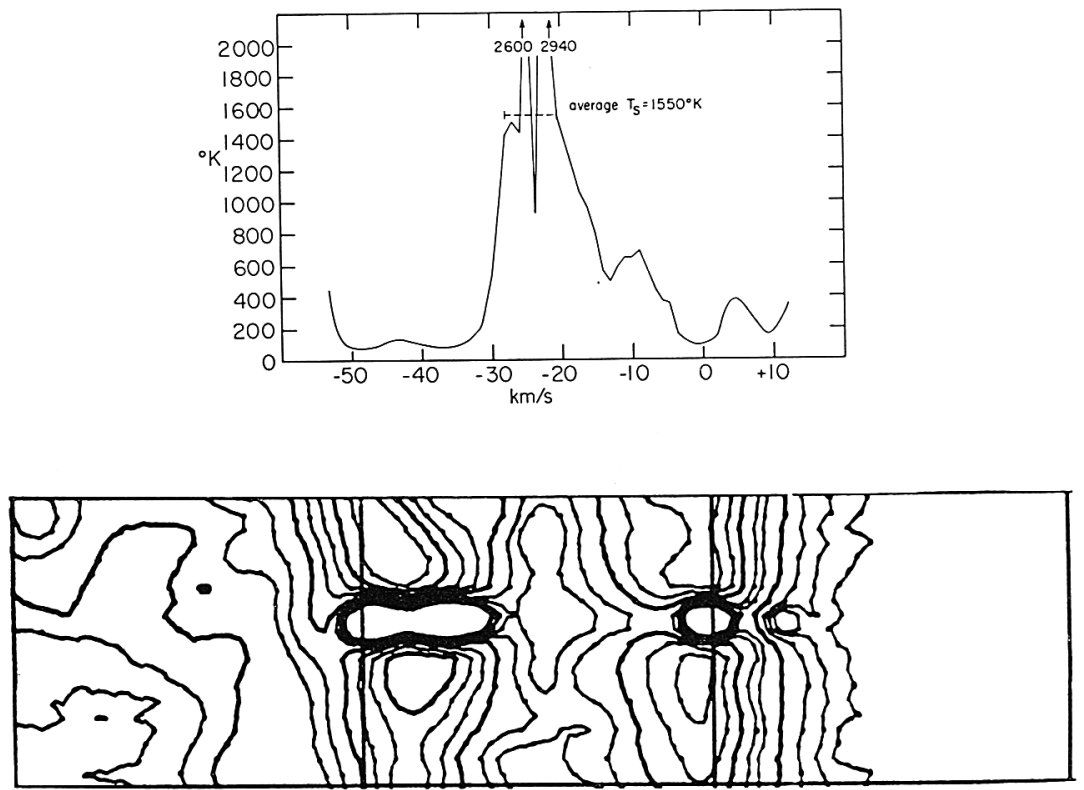

Fig. 6. Temperatures of concentrations of Hr producing absorption in Cas A as determined by Williams. Lower figure: enlarged portion of Cas A absorption. (Taken from Figure 5.) Upper figure: plot of observed $T_{\mathrm{ex}}$ values of features shown in the lower map.

assumed universal value of $125 \mathrm{~K}$. In their discussion Shuter and Verschuur showed that in absorption, cold clouds are far more visible than hot clouds. The distribution of cloud temperatures they found was therefore biased. To reproduce the then-accepted value of $125 \mathrm{~K}$, Shuter and Verschuur had to add to their observed distribution of cloud temperatures about 20 percent more clouds with temperatures $>120 \mathrm{~K}$. Presumably, these hotter clouds had been missed in the absorption studies.

Clark (1965), in discussing his interferometer measurements of $\mathrm{HI}_{\mathrm{I}}$ absorption lines, proposed an alternative to the isolated cloud model in terms of which earlier investigators had tended to interpret their results. Clark wrote: “... it is worthwhile to at least examine another alternative to this cloud explanation. This is to assume that the hydrogen seen in emission is not entirely the same hydrogen as that seen in absorption. This can come about when part of the hydrogen is at very high temperature, and thus has a very low absorption coefficient. In order to make it invisible in the absorption spectrum, it must have a temperature in excess of $1000 \mathrm{~K}$. Let us now assume that this hot hydrogen is a continuous, hot medium surrounding clouds somewhat cooler than $100 \mathrm{~K}$."

Clark supported his model by a variety of observational evidence involving both velocity and temperature measurements. The Clark model does indeed appear to be of the correct character. High temperatures have recently been measured in the ambient interstellar gas by several observers; for example, by Mebold (1969), by Radha- 
krishnan and Murray (1969), and by Williams (1969). There can be little doubt that the interstellar medium is a mixture of hot gas and embedded cold regions. Radhakrishnan and Murray speak of such a mixture as a 'raisin pudding' model of the interstellar medium.

Firm knowledge of the properties of the 'raisin pudding' is seriously lacking; observational clarification of the characteristics of the interstellar medium is crucial if proper theoretical insights are to be gained. Questions that require answers are, for example:

(1) Is the enveloping gas (the 'pudding') distributed on the large scale more or less smoothly in $R$ and $z$, with concentrations of cold hydrogen (the 'raisins') being everywhere present except that they are far more numerous in the spiral arms than elsewhere, or

(2) Are the arm and arm-like structures composed of generally cool gas in which still cooler concentrations of hydrogen exist, with the cool, large-scale arm structures being surrounded by hot gas which, certainly in the interarm regions, and possibly elsewhere, reaches temperatures greater than $1000 \mathrm{~K}$ ?

(3) What is the nature of the boundary of a concentration of cool gas in the arms? Is the boundary between the hot surrounding medium and the cool concentration sharply defined, a narrow transition zone between two phases of the gas, or

(4) Are there, in relatively cool arms of the Galaxy, temperature and density fluctuations associated with a turbulence spectrum?

(The question of the sharpness of the edge of concentrations of cool gas is crucial. A strong answer to this question might well establish the class of physical mechanisms responsible for formation of the concentrations.)

These questions which arise immediately in connection with a description of the interstellar gas, lead, of course, to more basic and fundamental questions of what causes the cool gas to be concentrated in spiral structures - spiral structures which appear to differ in overall character and not just in density of material from the surrounding interarm gas. Specifically, such questions relate to the principal problems involving current theory and observations of the interstellar gas. An important part of our discussion should determine how current observations fit into the two-phase model of the interstellar gas (Goldsmith et al., 1969), the density-wave model of spiral structure (Lin, 1967), and Roberts' $(1969,1970)$ discussion of density-wave induced shock phenomena in the gas on the inside edge of spiral arms.

\section{SOME CHARACTERISTICS OF THE GAS CONCENTRATIONS WITHIN SPIRAL ARMS}

The patchy, sharp-edged appearance of obscuring clouds of dark material seen so frequently on photographs of the Milky Way combined with the sharpness of the multiple interstellar lines seen in many stellar spectra no doubt gave rise to the hypothesis that the interstellar medium is composed quite generally of discrete spherical 'clouds' to which, as a first approximation, were assigned standard size, mass, density, and so forth. The numerous and still rapidly accumulating observations of neutral hydrogen do not lend support to such a hypothesis of isolated clouds of standard 
properties. The $\mathrm{HI}_{\mathrm{I}}$ gas, as described earlier, appears, on the galactic scale, to be concentrated in very large structures - spiral arms, spurs, interarm links. Within these major, large-scale structures there appears to be a continuum of gas in which there are smaller-scale concentrations having a variety of characteristics.

The picture of a wide variety of concentrations in a continuum of neutral hydrogen which formed a major structural unit in the Galaxy was first clearly brought to view by Heiles (1967a), who used the 300-foot antenna at Green Bank to investigate the region $l=100^{\circ}$ to $140^{\circ}, b=+13^{\circ}$ to $+17^{\circ}$. The hydrogen he observed constitutes a portion of the local spur in which the Sun is located. Broadly, Heiles found that in the area he investigated about three-fourths of the total $21-\mathrm{cm}$ emission came from a generally smooth background, the ubiquitous gas surrounding the Sun. Within that background Heiles found what he describes as two sheets of gas, that is, concentrations of hydrogen with different mean velocities and small velocity dispersions, and with continuity of density and mean velocity over large angular extent, particularly in longitude. Such sheet-like structure may or may not be common or frequent; that aspect of the picture given by Heiles is not of primary concern in this discussion. Our interest centers on structures of smaller scale than the two sheets of gas. Within the sheets of gas, Heiles found smaller-scale concentrations of several types:

(1) a few concentrations of gas generally resembling 'standard' clouds having characteristic radii of $10 \mathrm{pc}$, number densities of $6 \mathrm{H}$ atom ${ }^{-3}$, and masses $\approx 10^{3} M_{\odot}$;

(2) a small number of concentrations of larger dimensions, characteristically $20 \mathrm{pc}$ radii, number densities $\approx 2 \mathrm{H}$ atom $\mathrm{cm}^{-3}$, and masses of the order of a few $\times 10^{3}$ $M_{\odot} ;$ and

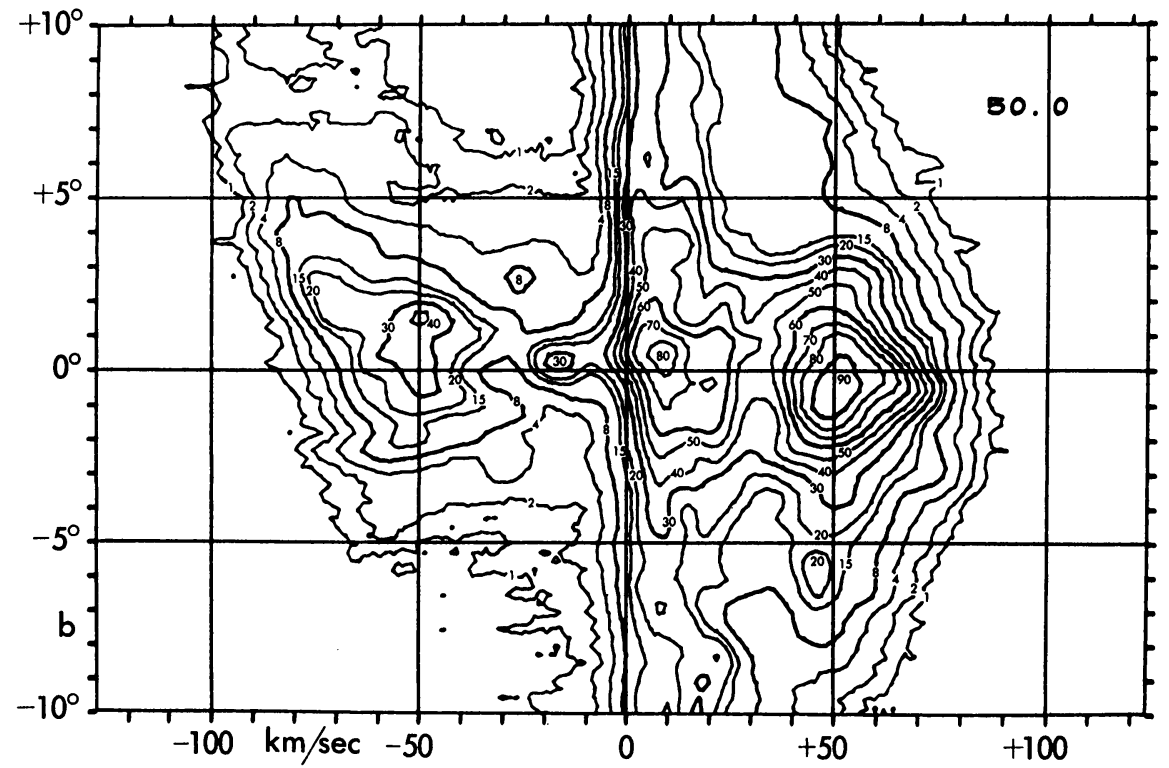

Fig. 7. Contour map $T_{A}\left(v_{r}, b \mid l=50^{\circ}\right)$ from the Hat Creek Survey of HI. 
(3) very many 'cloudlets' having, at the mode, radii of $2 \mathrm{pc}$, number densities of $2 \mathrm{H}$ atom $\mathrm{cm}^{-3}$, and masses $\approx 4 M_{\odot}$.

The Hat Creek observations (Weaver and Williams, 1970) can be used to test Heiles' hypothesis of many concentrations or density fluctuations in a continuous medium. The test area arbitrarily selected for this test extends from $l=44^{\circ}$ to $54^{\circ}, b=-4^{\circ}$ to $-10^{\circ}$. An observed contour map, $T_{A}\left(v_{r}, b \mid l=50^{\circ}\right)$, Figure 7 , will serve to characterize the general area; we look through the local gas to more distant spiral features. A set of closely spaced contour maps, $T_{A}\left(l, b \mid v_{r}\right)$, Figure 8 shows, on the sky, the hydrogen intensity in a series of narrow velocity ranges. These $T_{A}\left(l, b \mid v_{r}\right)$ maps are, essentially, pictures of the hydrogen brightness in a narrow distance range at fixed distance from the Sun. Examination of a velocity sequence of such maps shows clearly (a) the existence of numerous concentrations or
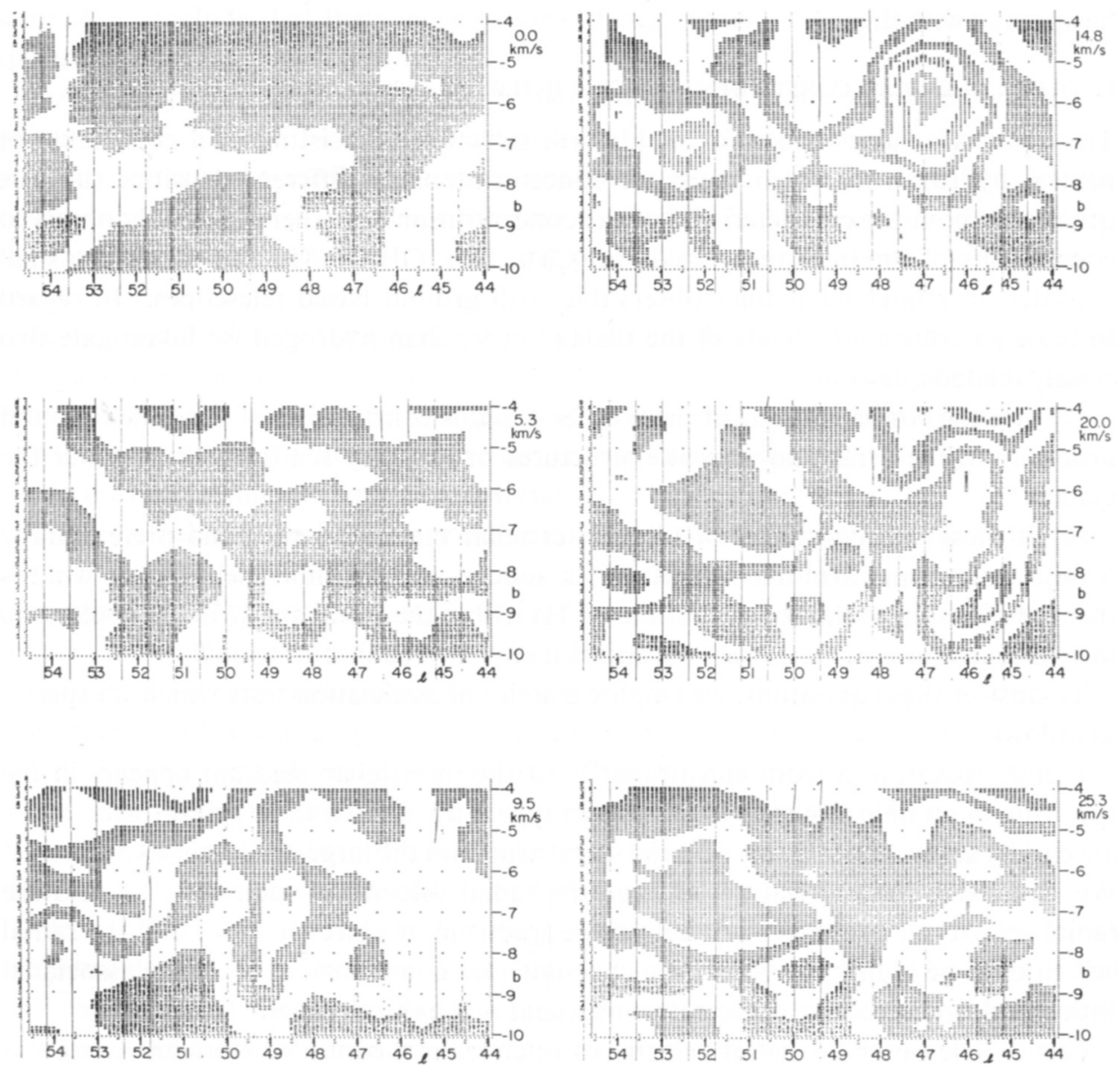

Fig. 8. Set of $T_{A}\left(l, b \mid v_{r}\right)$ maps for ranges $l=44^{\circ}$ to $54^{\circ} .5, b=-4^{\circ}$ to $-10^{\circ}$ at the velocity values shown in the diagram. The velocity range covered in each diagram is $2.11 \mathrm{~km} \mathrm{sec}^{-1}$. 
'cloudlets' of small angular extent and narrow velocity dispersion, and (b) a variety of gas concentrations of larger angular size but small velocity dispersion.

The numerical data for these various concentrations or density fluctuations are in excellent agreement with the data tabulated by Heiles. For examples, we find (Figure 8) for the largest concentration centered at $l=47^{\circ}, b=-6^{\circ}, v=14.8 \mathrm{~km} \mathrm{sec}^{-1}$, assumed distance $=750 \mathrm{pc}$ :

$$
\begin{aligned}
& \text { radius } \approx 22 \mathrm{pc} \\
& \left\langle n_{\mathrm{H}}\right\rangle \approx 5 \text { atom cm} \\
& \text { mass } \approx 4500 \mathrm{M}_{\odot} \\
& \sigma_{v} \approx 3.2 \mathrm{~km} \mathrm{sec}^{-1} .
\end{aligned}
$$

The general background in which the concentration occurs is 0.5 to $1.0 \mathrm{H}$ atom $\mathrm{cm}^{-3}$. The concentration of gas at $l=47^{\circ}, b=-6^{\circ}, v=14.8 \mathrm{~km} \mathrm{sec}^{-1}$ is not gravitationally bound. It, like the other gas concentrations pictured, must form and 'evaporate' with time.

\section{E. OTHER CHEMICAL CONSTITUENTS OF THE INTERSTELLAR MEDIUM}

The observational evidence for major arm structures consisting of a continuum of neutral hydrogen in which there are concentrations of different densities suggests questions about the characteristics of the concentrations of other commonly observed constituents of the interstellar medium: CaII, $\mathrm{NaI}, \mathrm{OH}, \ldots$. (In this review we shall consider only interstellar lines observable with ground based telescopes.) In regard to these gaseous constituents of the Galaxy other than hydrogen we investigate two closely related questions:

(1) Are the concentrations of these other gaseous constituents of the Galaxy found primarily in the arms and arm-like structures or are they scattered throughout the Galaxy?

(2) If these concentrations of other interstellar materials are found preferentially within the arm and arm-like structures (as, indeed, we shall find them to be), what is their relationship to the concentrations of $\mathrm{HI}$ within the spiral arms? In particular, are the concentrations of $\mathrm{Ca}$ II, $\mathrm{Na}$ I, ..., coincident with $\mathrm{HI}$ concentrations?

To answer these questions, we employ a series of association tests which we specify as follows.

Test 1. Absorption from constituent A of the interstellar medium appears in the spectrum of an object of type B. Objects of type B are known to be spiral tracers; they are closely associated in space with the spiral arms and the large-scale arm-like features. We investigate the correlation between the radial velocity of each object $B$ and the radial velocity of constituent $A$ seen in the spectrum of object $B$. If we find the radial velocities to be highly correlated, we conclude that constituent $A$ is closely associated with spiral structure; like objects $B$, constituent $A$ is a spiral tracer.

Test 2. The observed radial velocity of interstellar medium constituent $A$, seen in direction $\alpha$, is compared with the expected velocity of objects of class B in direction $\alpha$. Class B objects are known spiral tracers. If we find a high positive correlation between 
the values compared, taking into account the known dispersion in the expected velocity of objects $B$ and the error of measurement of the velocity of $A$, we conclude that constituent $\mathrm{A}$ is a spiral tracer also.

Test 3. Absorption lines from chemical constituents $\mathrm{A}$ and $\mathrm{B}$ of the interstellar medium appear in the spectrum of object $C$, which serves merely as a source of continuum radiation. If the observed radial velocities of constituents $A$ and $B$ are the same within measuring error, we assume that the concentrations of constituents $A$ and $B$ are coincident in space. As a secondary criterion of coincidence, we should find similar profiles for the lines arising from constituents $A$ and $B$ if the materials are coexistent and well mixed in a single concentration in space.

Test 4. We observe that spectral lines from constituents A and B of the interstellar medium are observed in one and the same precisely defined area of the sky and are not found outside of that area. The velocities of A and B are found to be the same within measuring error. Again, but of secondary character, we find the line shapes to be the same. We conclude that the concentrations of constituents A and B from which the spectral lines arise are coincident in space.

Test 5. A spectral line arising from constituent A of the interstellar medium is observed over an area of the sky identical with that occupied by some visible constituent B of the interstellar medium (a dark obscuring cloud, for example). We do not observe the spectrum of A outside the area covered by $\mathrm{B}$. We conclude that the concentration constituents $\mathrm{A}$ and $\mathrm{B}$ responsible for the features observed are coexistent in space.

Tests 1 and 2 relate to the establishment of certain observed gaseous constituents of the Galaxy as spiral tracers; Tests 3, 4, and 5 relate to the establishment of coincidence of different gaseous constituents within a single concentration in space.

We consider first the question: are the concentrations of interstellar gas that give rise to the optical interstellar lines spiral tracers?

The close association between spiral arms as defined by $\mathrm{HI}$ and the gas concentrations giving rise to the strongest and most frequently seen interstellar lines (those of $\mathrm{Ca} I \mathrm{I}$ and $\mathrm{NaI}$ ) is clearly demonstrated by Münch's (1965) diagram showing the observed relation between radial velocity of interstellar $\mathrm{Ca}$ II and/or $\mathrm{NaI}$ and galactic longitude. To a very high degree the observed velocity-longitude for the interstellar lines agrees with the analogous velocity-longitude diagram found for $\mathrm{HI}$ by Weaver (1970). On the basis of Test 2, we conclude that the concentrations of $\mathrm{Ca}$ II and $\mathrm{NaI}$ from which originate the observed interstellar lines are closely associated with the concentrations of $\mathrm{HI}$ which define the gaseous spiral arms. Concentrations of interstellar gas which produce lines of $\mathrm{Ca}$ II and $\mathrm{Na}$ I are spiral tracers.

We consider Test 1, and, in Figure 9, illustrate the correlation between radial velocities derived from the interstellar lines of $\mathrm{CaII}$ and $\mathrm{NaI}$ and the velocities of the OB stars in which the interstellar lines are observed. The correlation is positive and high. Howard et al. (1963) found a correlation coefficient of +0.97 , while Takakubo (1967) found a correlation coefficient of +0.85 from studies of this kind. Münch (1965) pointed out that in some areas of the sky interstellar lines show a systematically nega- 


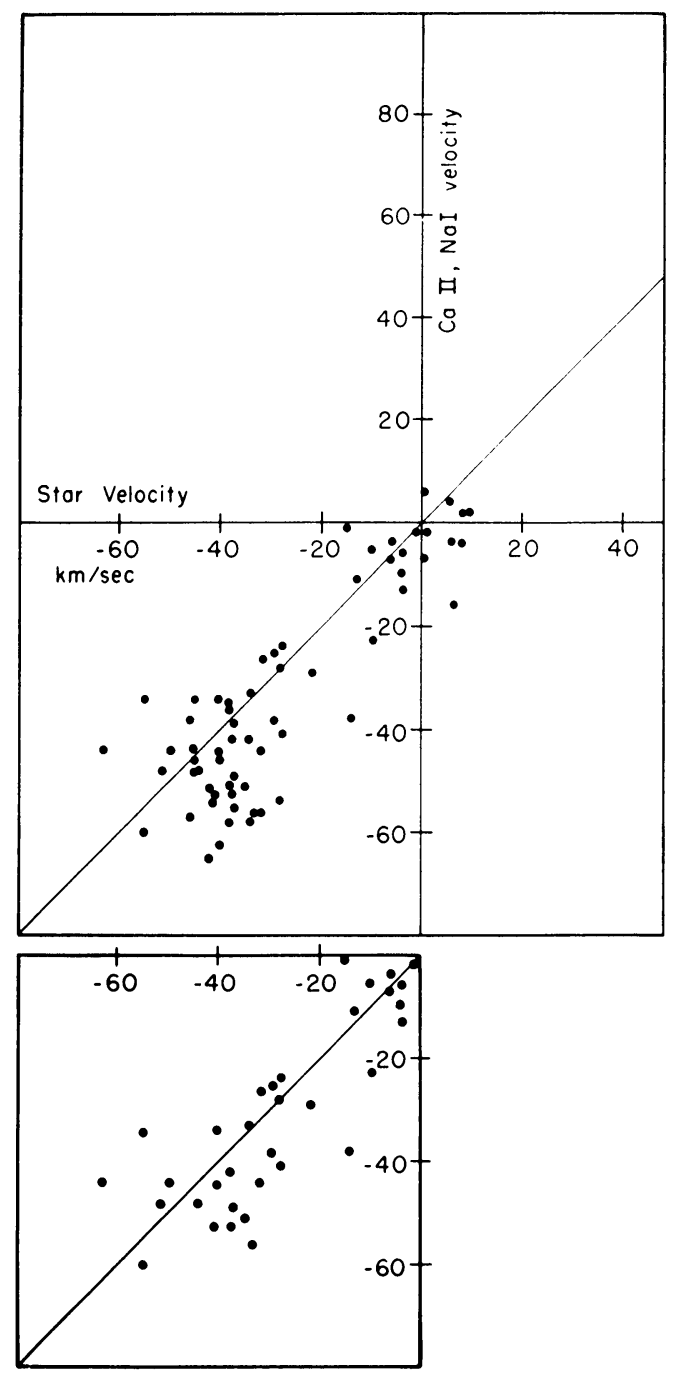

Fig. 9. The relationship between velocities of interstellar CaII and the B stars in whose spectra the interstellar lines appear. The lower diagram is the same as a section of the upper one except for the omission of a group of stars from a region in the sky known to show systematically negative velocities.

The larger-than-normal negative velocities of the CaII lines shown by the omitted stars is readily apparent.

tive radial velocity with respect to the associated stellar radial velocities. The explanation proposed by Münch is that the interstellar gas, which lies between the stars and the observer is, in the part of the Galaxy affected, systematically expanding away from the group of stars involved. Such an effect, while interesting and, for some purposes, of dominant importance, does not affect our conclusion, namely that the high positive value of the correlation coefficient represented by Figure 9 (and found numerically by 
Howard et al. and by Takakubo) clearly indicates the close association between the $\mathrm{OB}$ stars and the concentrations of gas giving rise to the interstellar $\mathrm{Ca}$ II and $\mathrm{Na}$ I lines.

Interstellar absorption lines in the optical range observable from the ground have been identified from NaI, KI, CaI, CaII, TiII, FeI, $\mathrm{CH}, \mathrm{CH}^{+}$, and $\mathrm{CN}$. Concentrations of interstellar gas contain a variety of materials; visibility of particular spectral lines depends upon the atomic or molecular constants of the materials present and upon the physical conditions within each gas concentration. We test the hypotheses that the various interstellar materials identified by their spectral lines in a single star arise from a single concentration of gas. We employ Test 3 and compare radial velocities derived from the interstellar lines. Invariably, we find that the various lines may be grouped together on the basis of agreement of radial velocity. All lines originating in one gas concentration show the same radial velocity within the limits of measuring accuracy. We take as an example Herbig's (1968) observations of $\zeta$ Oph, which contains in its spectrum interstellar lines from more than one gas concentration. In one concentration of gas, the star exhibits all identified optical interstellar molecules and atoms except Tili; from a second gas concentration only a few lines are seen. For each concentration the lines agree in radial velocity within measuring errors. Test 3 leads us to conclude that all lines of one velocity arise in one concentration of gas as, indeed, Herbig assumed in his analysis.

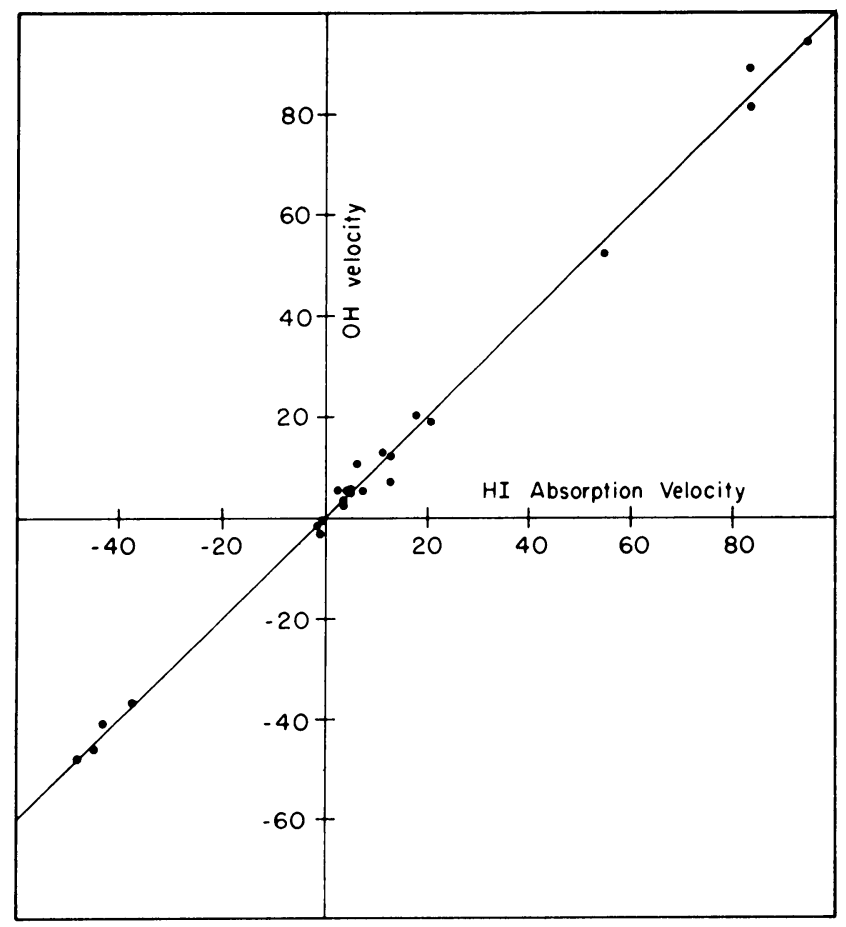

Fig. 10. Correlation diagram of the velocity of $\mathrm{OH}$ absorption and $\mathrm{HI}$ absorption observed in one and the same source. 
Interstellar lines arising from constituents other than $\mathrm{CaII}$ and $\mathrm{NaI}$ can always be associated in a gas concentration of $\mathrm{CaII}$ and $\mathrm{NaI}$ on the basis of velocity. Our tests have shown that gas concentrations from which $\mathrm{Ca}$ II and $\mathrm{Na}$ I lines originate are spiral tracers; we therefore conclude that all interstellar optical lines originate from gas concentrations that are spiral tracers.

The $\mathrm{OH}$ molecule is seen in many radio sources distributed around the galactic plane In Figure 10 we apply Test 3 to the radial velocities derived from the absorption lines of $\mathrm{OH}$ and $\mathrm{HI}$ seen in the same source. The data for the comparison are from Goss (1968). Within measuring accuracy the velocity relationship is one to one; the correlation coefficient approaches unity. We conclude that the concentrations of absorption of $\mathrm{OH}$ and $\mathrm{HI}$ are spatially coexistent; the $\mathrm{OH}$ is as much a spiral tracer as the $\mathrm{HI}$.

$\mathrm{OH}$ emission lines originate in sources of several types.

(1) On the basis of Test 5 Heiles (1968) concluded that there is 'normal' OH emission (presumably $\mathrm{OH}$ in thermodynamic equilibrium) associated with dark obscuring clouds. The dark clouds are known spiral tracers; the $\mathrm{OH}$ producing the emission is thus closely connected with spiral arms.

(2) Anomalous, highly non-thermal $\mathrm{OH}$ emission, generally strong in the principal lines, arises from concentrations of gas of very small physical size which a modified Test 5 shows to be associated with some Hil regions. (See, for example, Weaver et al., 1968.) Since $\mathrm{HII}$ regions are prime spiral tracers, the $\mathrm{OH}$ gas again is found to be associated with the spiral arms.

(3) Anomalous non-thermal $\mathrm{OH}$ emission, often mainly visible in the satellite lines, frequently appears to be associated with non-thermal radio sources. (See Turner, 1969.) Test 2 leads to the hypothesis that the $\mathrm{OH}$ from which the lines originate is involved in the spiral arms.

(4) Anomalous $\mathrm{OH}$ absorption, mainly in the $1612 \mathrm{MHz}$ satellite line appears to be associated with some infrared stars (Wilson and Barrett, 1968). Since this is a stellar phenomenon rather than an interstellar one, we do not consider it further.

The radio lines of $\mathrm{H}_{2} \mathrm{O}$, like those of one of the classes of $\mathrm{OH}$, appear to be closely associated with Hil regions. (See, for example, Cheung et al., 1969b; Knowles et al., 1969; and Meeks et al., 1969). Further, again like $\mathrm{OH}, \mathrm{H}_{2} \mathrm{O}$ is strongly non-thermal, shows maser action, and exhibits pronounced variations in intensity with time. The close association between the $\mathrm{H}_{2} \mathrm{O}$ and $\mathrm{H}$ II regions indicates location of the gas concentrations showing $\mathrm{H}_{2} \mathrm{O}$ in spiral arms.

$\mathrm{H}_{2} \mathrm{CO}$ is rather widely observed along the galactic plane. (See Snyder et al., 1969; Palmer et al., 1969; Rydbeck et al., 1969.) $\mathrm{HI}, \mathrm{OH}$, and $\mathrm{H}_{2} \mathrm{CO}$ are often seen together in absorption (though they are not necessarily, of course, all seen in each source); $\mathrm{H}_{2} \mathrm{CO}$ is observed in some dark clouds in which 'normal' $\mathrm{OH}$ emission is present. Application of Test 3 leads to the conclusion that the associated lines arise from materials located in single concentrations of gas. Test 4 indicates that in some instances the various constituents are differently localized within the gas concentrations. From each test, however, we reach the strong conclusion that the $\mathrm{H}_{2} \mathrm{CO}$ is found in concentrations of gas closely associated with spiral arms. 
To date, $\mathrm{NH}_{3}$ has not been found widely distributed. (See Cheung et al., 1968; Cheung et al., 1969a, c.) This may be instrumental rather than physical; very low noise receivers may be required for extensive discovery of $\mathrm{NH}_{3}$ sources. Tests 3 and 4 lead to the conclusion that in the places in which $\mathrm{NH}_{3}$ has been observed, it is closely related to $\mathrm{OH}$ absorption which we associate with gas concentrations in spiral arms.

We sum up these various tests by noting simply that the concentrations of gas which give rise to the interstellar lines, both optical and radio, appear to be constituents of the spiral arms of the Galaxy. More specifically, the various constituents producing interstellar lines in the optical range appear to exist together in single condensations as do those producing radio lines. In the next section we examine further relations between the condensations of gas.

F. SOME INTERRELATIONS BETWEEN OBSERVED ATOMIC AND MOLECULAR CONSTITUENTS AND PHYSICAL PROPERTIES OF INTERSTELLAR CONCENTRATIONS OF GAS

An arm of the Galaxy appears to be composed of a continuum of gas in which concentrations of cooler gas are present. The concentrations of $\mathrm{H}$ i seen in emission exhibit a variety of sizes and masses. The study by Heiles (1967) of an area of 160 square degrees is currently the only one which directly provides fairly extensive quantitative data on size and mass of the concentrations of interstellar gas. Heiles found only a few large concentrations that generally resemble the 'standard' clouds which have been widely discussed in the literature; he observed very many small concentrations of gas which he termed 'cloudlets'. In Figures 11 and 12 we reproduce the distributions of radii and masses found by Heiles for the 'cloudlets' in the area he investigated. Heiles states that the finite angular resolution of the telescope used for the observations does not distort the distribution of radii found; the instrumental angular resolution corresponds to $R=0.75 \mathrm{pc}$ at the average distance of the concentration observed. This value lies on the far left side of the published distribution of $R$-values.

The concentrations of interstellar gas in which absorption lines originate exhibit the same spatial distribution as the concentrations of $\mathrm{H}_{\mathrm{I}}$ that are seen in emission, and that define the gas arms of the Galaxy. The concentrations of gas in which absorption lines originate are mixtures of atomic and molecular materials; they, like the emitting H I concentrations appear to exhibit a variety of different sizes, densities, and temperatures, though few quantitative data are available. Which particular absorption lines are seen in any concentration depends upon the spectroscopic properties of the atomic and molecular species present and upon the physical conditions within the gas concentration.

In the optical range of the spectrum mainly the strong lines of $\mathrm{Ca}$ II and $\mathrm{NaI}$ are observed in the interstellar gas concentrations, but, at the same time, it is not uncommon to find, originating from a concentration of gas which shows $\mathrm{Ca}$ II and $\mathrm{NaI}$, lines from a variety of other atoms and molecules as well. Indeed, some single concentrations exhibit all identified optical interstellar lines.

In the radio range, absorption lines of various materials likewise originate in a single concentration of gas. $\mathrm{HI}_{\mathrm{I}}$ is often seen together with the molecules $\mathrm{OH}$ and 


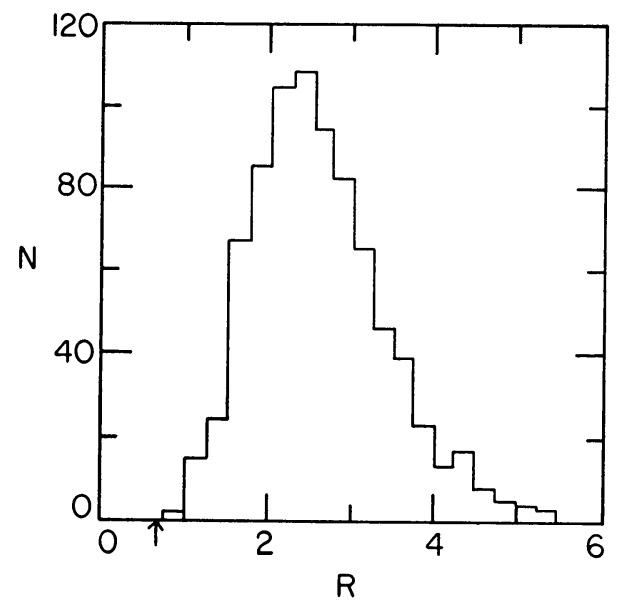

Fig. 11. Distribution of radii of cloudlets. (From Heiles, 1967a.) Note the position of the arrow on the abscissa scale. The $R$-value indicated by the arrow represents the resolving power of the telescope used for the observations.

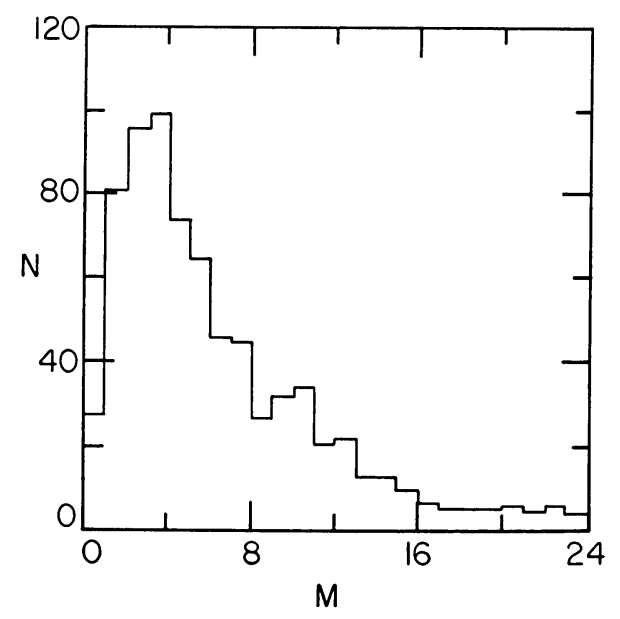

Fig. 12. Distribution of masses of cloudlets. (From Heiles, 1967a.)

$\mathrm{H}_{2} \mathrm{CO}$, all having origin in a single gas concentration. They are not, of course, seen together in every source; various combinations occur, presumably because of physical conditions in the gas concentration. Anomalous emission from $\mathrm{OH}$ and $\mathrm{H}_{2} \mathrm{O}$ appear to come from sources of very small size associated with $\mathrm{H}$ II regions in special situations. Absorption lines of $\mathrm{OH}$ and $\mathrm{NH}_{3}$ appear together in a few sources; $\mathrm{NH}_{3}$ is not widely observed. In some dark obscuring clouds of particulate material that strongly attenuates optical radiation, 'normal' $\mathrm{OH}$ emission is observed. It is possible that all such clouds are emitting and that the emission would be found if very low noise receivers were available for the observations. Some dark clouds show $\mathrm{H}_{2} \mathrm{CO}$ in absorption. Several investigators have found the dark obscuring clouds to be highly 
deficient in H I 21-cm radiation; see Heiles, 1967a, b; Kerr and Garzoli, 1968; Varsavsky, 1968; and Mészáros, 1968. The most complete investigation is the recent one by Heiles (1969), who surveyed 48 dark clouds in Hi emission. He found only one cloud that showed excess $\mathrm{HI}$, and its $35 \mathrm{~K}$ emission he assumed to be saturated. Two clouds showed deficiencies in $\mathrm{HI}$, while another showed a complex structure not immediately interpretable. All the rest ( 44 objects) did not show H I radiation.

A directly related observation of a broader character is the ratio of $\mathrm{HI}$ emission to dust, which has been studied by a number of investigators. Bok et al. (1955) early demonstrated that the $\mathrm{HI} /$ dust ratio decreased in the direction of dark clouds. (See also Garzoli and Varsavsky, 1966.) Pronik (1963) showed that for the Omega Nebula the $\mathrm{HI} /$ dust ratio decreased from normal by an order of magnitude.

It is reasonable to assume that in the dark clouds that have been observed, the abundances of the elements is reasonably normal (at least in general order of magnitude) and that physical conditions in the gas are primarily responsible for what spectral lines are observed. One may suppose, with Heiles and other investigators, that in the very dark clouds cooling has gone on to such an extent that, while some molecules are present and are obscured, the gas that would normally be seen as $\mathrm{HI}$ has been frozen down to $\mathrm{H}_{2}$, hydrogen mantles on grains, or to some other form that eliminates it as a candidate for observation as $\mathrm{HI}$.

The concentrations of gas in the arms of the Galaxy thus show a wide range of temperatures. At the low end of the temperature range are the dark clouds of particulate material in which molecules are observed but in which atomic hydrogen is not seen, and is presumably frozen down. At the high end of the temperature range are concentrations of gas showing $\mathrm{HI}$ excitation temperatures of a few hundred degrees. Preliminary observations indicate that these 'hot' concentrations do not show molecular lines. It is not known with any precision how the temperatures of the concentrations are distributed within this range of temperatures, though gas concentrations are observed throughout the whole temperature range. Different observing techniques emphasize different portions of the range. It is not known where the high-temperature cutoff for concentrations of gas occurs; it does appear that there is gas in the interarm region for which the excitation temperature is $>1000 \mathrm{~K}$. There is, however, no indication that the interarm region contains concentrations of gas of the character observed to occur in large numbers in the arms of the Galaxy. Direct observational evidence relating to the temperature of the intercloud gas in the arms, that is, the continuum of gas in which the concentrations are located, is lacking.

Hil regions show both optical and radio lines in emission. Can we find an $\mathrm{HI}$ region - that is, a single concentration of cool gas in an arm of the Galaxy - which shows both optical and radio lines? The optical lines would be seen as interstellar absorption lines. Hobbs (1969a, b) has shown that the $\mathrm{NaI}$ lines he measured are formed in $\mathrm{HI}$ regions; we expect such a situation to be true in general for optical interstellar absorption lines.) The radio lines from the $\mathrm{H}$ I region could be seen either in emission ( $\mathrm{HI}$, for example) or in absorption. Various investigators have stated that they have found equivalence between optical and radio lines (e.g., Herbig, 1968; 
Riegel and Jennings, 1969), but the velocities of optical and radio lines discussed differ by one or two (or more) $\mathrm{km} \mathrm{sec}^{-1}$. Such a difference is greater than can be accepted for the association tests applied earlier, and does not constitute what would here be looked upon as proof of spatial coexistence of the radio and optical sources. We do not consider in this context agreement between an optical line velocity and the velocity from a 'gaussian component' of $\mathrm{HI}$ as indication of coexistence.

The most extensive test for spatial coexistence of radio and optical interstellar line sources was made by Habing $(1969 \mathrm{a}, \mathrm{b})$. He investigated 48 cases, conclusively proving that the concentrations from which the optical interstellar lines originate are spiral tracers. (Essentially he used Test 2.) However, he found no normal case in which there is agreement (within observational errors) of optical interstellar velocity and the velocity of a specific $\mathrm{HI}$ concentration. (We here omit consideration of two highvelocity cloud cases which might be abnormal.) The lack of precise agreement between velocities derived from optical and radio interstellar lines in H I regions is surprising; its significance requires clarification.

\section{G. VELOCITY DISPERSION AMONG SUB-UNITS OF AN ARM AND IN THE GAS BETWEEN CONCENTRATIONS}

In discussing velocity dispersion, one must be specific as to the type of unit or subunit of the Galaxy that is under discussion. In the past, on the basis of the standard cloud model of the Galaxy, reference has been made to 'internal dispersion' and 'external dispersion', that is, dispersion of velocities within a cloud or among clouds. The gaseous medium between the clouds was not considered, nor was the medium between arms. One might therefore wish to add other dispersions in a compilation as, for example, a dispersion estimate relating to the intercloud gas - in the terms we have used in this review, the gas between concentrations - in an arm, or a dispersion estimate relating to the gas in the interarm region.

Van Woerden (1967) has provided useful tabulations of many published values of internal dispersions and external dispersions which, in this discussion will, for precision, be termed 'dispersion within concentrations' and 'dispersion among concentrations'. We shall find, unfortunately, that observational data for any category of structure are not numerous and that much observational work remains to be done.

(1) It is probable that the dispersion within H I concentrations is related to the size of the concentration. Thus, in the Hi in our local spur, Heiles (1967) finds:

$$
\begin{array}{ll}
\text { for 'cloudlets' } & \sigma=0.6 \text { to } 1.2 \mathrm{~km} \mathrm{sec}^{-1}, \\
\text { for 'larger concentrations' } \sigma \approx 0.9 \mathrm{~km} \mathrm{sec}^{-1}, & \begin{array}{l}
\sigma=1.2 \text { to } 4.5 \mathrm{~km} \mathrm{sec}^{-1}, \\
\text { mean } \sigma \approx 4.0 \mathrm{~km} \mathrm{sec}^{-1}
\end{array}
\end{array}
$$

These numerical values overlap older tabulated numerical values in which there has been mixing of a variety of types and sizes of concentrations located in many regions of the Galaxy. Quite frequently estimates of dispersion have been derived from analysis of profiles into gaussian components, little or no attention being given to the 
size or nature of gas structure. Caution is required if older values of dispersions are used.

Hobbs $(1969 \mathrm{a}, \mathrm{b})$ investigated many interstellar NaI lines with an interferometer having a bandpass at half-intensity of $0.51 \mathrm{~km} \mathrm{sec}^{-1}$. Many lines were found to be multiple. For 15 well-observed single lines Hobbs found values of $\sigma$ that ranged from 0.64 to $1.48 \mathrm{~km} \mathrm{sec}^{-1}$. If the kinetic temperature of the concentration of $\mathrm{Na}$ is $\leqslant 100 \mathrm{~K}$, thermal broadening of the lines is negligible and the measured velocity spread must represent turbulence almost completely. If, on the other hand, there is essentially no turbulence in the concentrations of $\mathrm{NaI}$, or CaII, the kinetic temperature of the concentrations must range from 1100 to $6100 \mathrm{~K}$. (If one observation is omitted, the upper limit becomes $4400 \mathrm{~K}$.)

The NaI lines observed by Hobbs and the HI 'cloudlets' observed by Heiles have similar dispersions. If the spectroscopic features reported originate in equivalent concentrations of gas, the gas temperature must be quite low. The observed dispersion of velocities would be representative largely of turbulence. However, the precise coincidence in space of, say, $\mathrm{Na}$ I and $\mathrm{HI}$ condensations remains an important question in need of further investigation.

(2) Very little numerical information on the dispersion among gas concentrations in major arms is available. Kerr (1964), observing HI with the 210-foot antenna in Australia, found, for the outer arm of the Galaxy, mean arm velocity changes of as much as $20 \mathrm{~km} \mathrm{sec}^{-1}$ in angular distances of $1^{\circ}$. This could indicate rather high dispersion values, at least in some volume elements in an arm.

Hat Creek observations of the velocities among gas concentrations in the major arms indicate that the velocity distribution may not be completely random. Rather, there appears a tendency for parts of arms to split in velocity by $10 \mathrm{~km} \mathrm{sec}^{-1}$ or more over some angular range, then to coalesce. Much material relating to this question is available; investigations are in progress.

Most data relevant to the dispersion of velocity among gas concentrations refer to the situation within the local spur. Blaauw (1952) investigated the K-line velocities of Ca II measured by Adams. Blaauw showed that an exponential law fits the observed distribution of velocities more closely than a gaussian distribution. From K-line velocities he derived a value of $\eta=5 \mathrm{~km} \mathrm{sec}^{-1}\left(\sigma=7 \mathrm{~km} \mathrm{sec}^{-1}\right)$ for the exponential distribution. The value found by Blaauw may represent an upper limit to the dispersion among the gas concentrations that give rise to optical lines; there is some question as to the value of the zero velocity with respect to which individual peculiar velocities should be measured. Takakubo (1967), using gaussian component analysis of the local $\mathrm{HI}$ as the basis of his discussion, found $\sigma=5.6 \mathrm{~km} \mathrm{sec}^{-1}$ and noted that this value changes with position on the sky and other properties of the population investigated. The physical significance of these variations is unclear.

(3) The dispersion of velocities frequently quoted for 'the motions of interstellar clouds' is $6 \mathrm{~km} \mathrm{sec}^{-1}$ (Westerhout, 1957). This value probably refers more nearly to the dispersion of the intercloud gas in the local spur than it does to the motions of concentrations of gas within the local spur. This frequently quoted value was deter- 
mined by Pottasch (see Westerhout, 1957), who fitted gaussian curves to the "forbidden velocities' (that is, the positive wings of the velocity distribution in the $l^{\mathrm{I}}$-range $60^{\circ}$ to $100^{\circ}$, latitudes $b^{\mathrm{I}}=0^{\circ}, \pm 2.5^{\circ}$ ) to find $\sigma=5.8$ to $9.7 \mathrm{~km} \mathrm{sec}^{-1}$, with $\langle\sigma\rangle \approx 6.0 \mathrm{~km} \mathrm{sec}^{-1}$. A similar mean value and range was found in other longitude zones.

In view of the differences in the characters of the gas structures and the natures of the motions investigated, there should be no surprise that Blaauw and Pottasch found different mathematical forms for the distributions they observed. Blaauw found an exponential law most satisfactory for representing the distribution of CaII velocities,
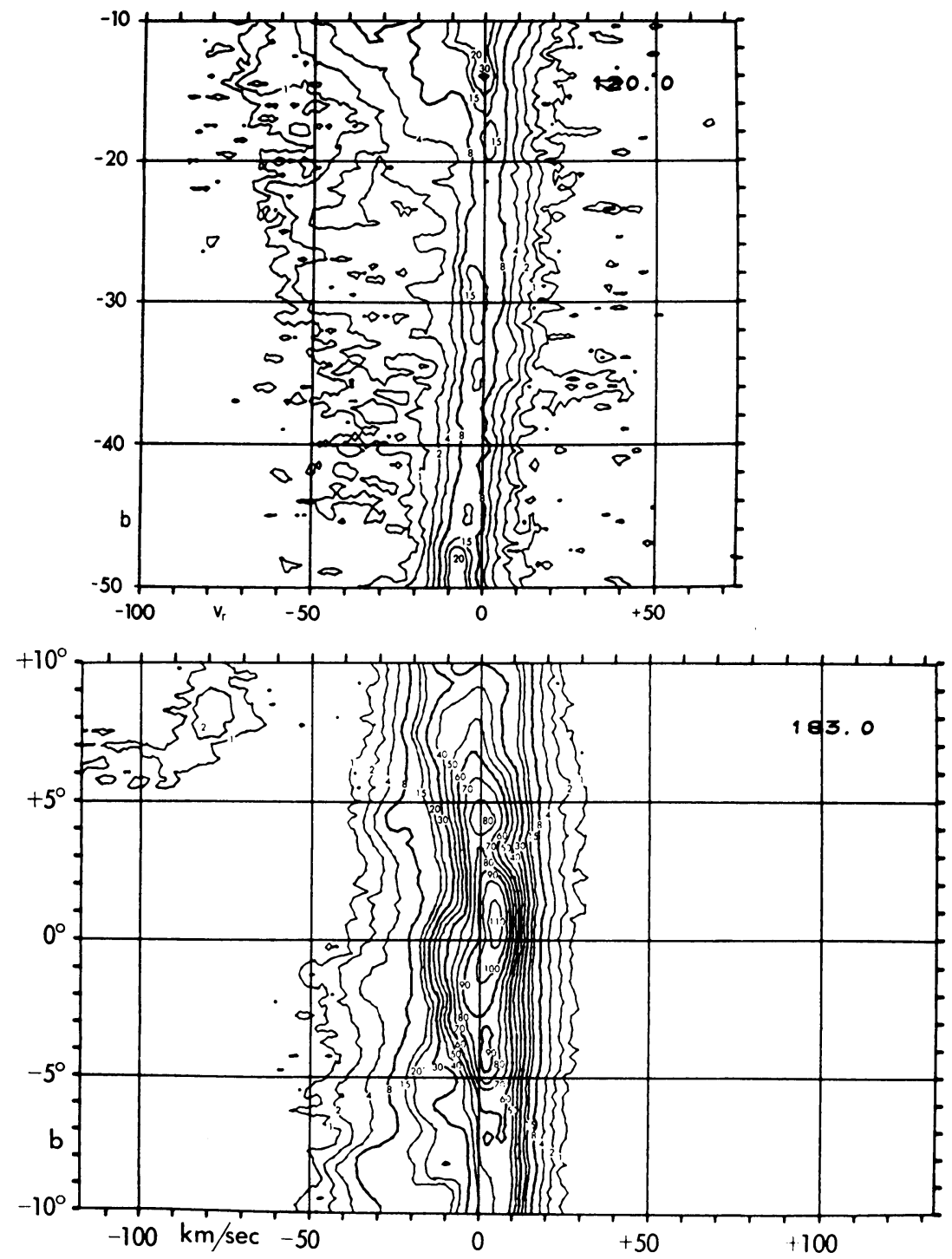

Fig. 13. Sample contour diagrams of $T_{A}\left(v_{r}, b \mid l\right)$ showing the preponderance of negative velocities among the low-intensity gas. 
while Pottasch determined that a gaussian function provided a better fit to the distribution of neutral hydrogen forbidden velocities.

(4) Systematic information relating to the velocity dispersion of the interarm gas is essentially lacking. From the scanty observational evidence a few trends may be visible.

(a) The dispersion velocity of the interarm gas is higher than that in the spiral arms, and may change from one part of the Galaxy to another in a random way.

(b) In the interarm region (probably more accurately in the region of an arm/
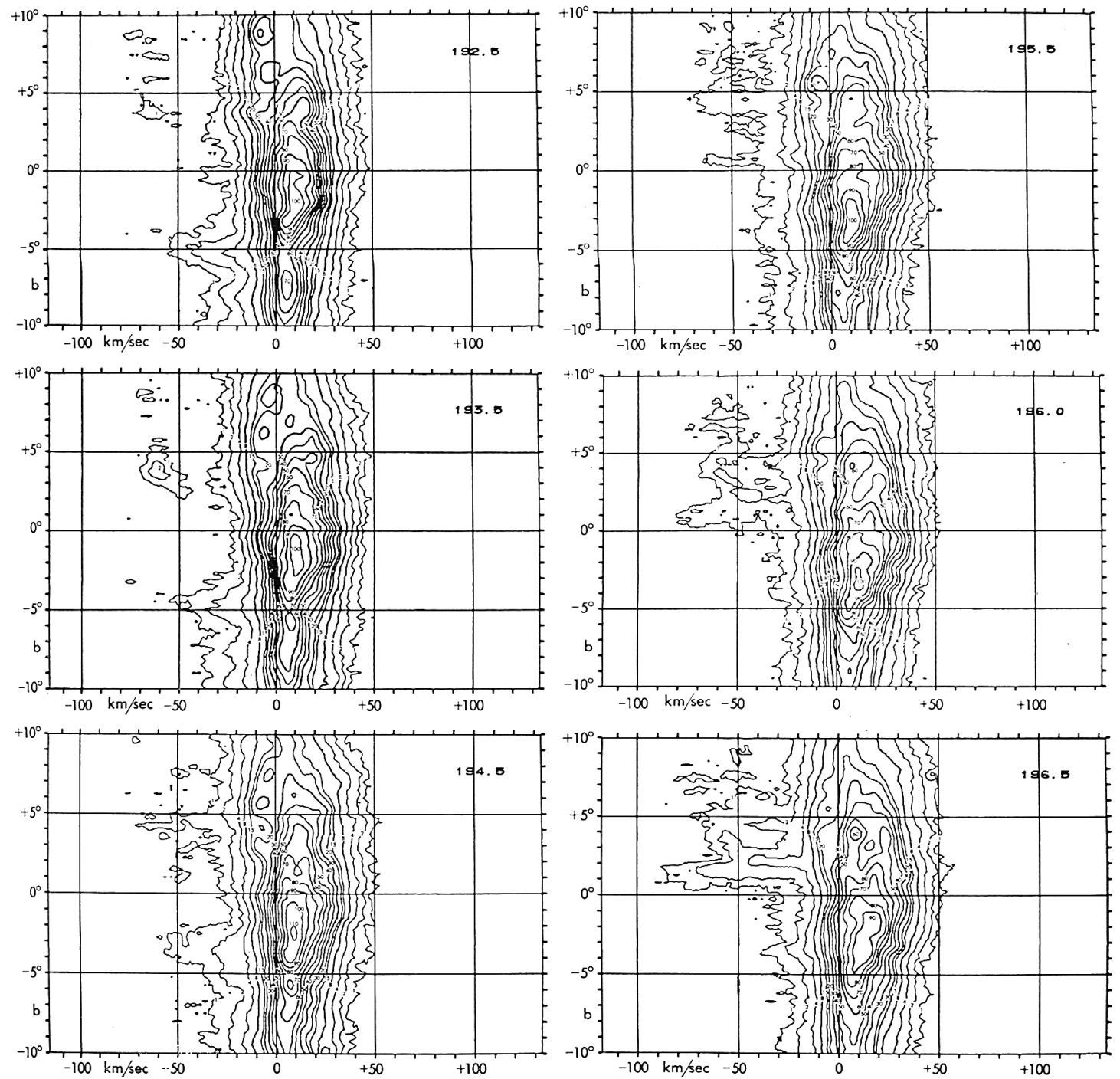

Fig. 14. Sample of contour diagrams illustrating the existence of 'active regions' in the Galaxy. Note the jet-like structures at longitude 196.5. 
interarm interface) the distribution of velocities may not be isotropic; there is normally a negative velocity tail.

The anisotropy of the observed distribution becomes a dominant factor as we go to higher velocities and lower intensities. Negative velocities are preponderant. The effect is not subtle and it must carry important information about the interstellar medium that we do not yet fully appreciate. Two contour maps (Figure 13) chosen almost at random will illustrate the point. The negative tails in the velocity distributions are very pronounced.

Discussion of negative velocities leads logically to the question of high-velocity gas and the problems related to its nature: is it galactic or extragalactic? Mrs. Dieter (1969) has recently reviewed the observational aspects of the high-velocity gas; her review is strongly recommended for further information on this subject. No attempt will be made in this review to present an abstract of her discussion. Rather, we conclude these remarks on the motion properties of the gas in the Galaxy with a few details of phenomena that should be of particular interest to members of this conference.

Most studies of high-velocity gas have been in latitudes $>15^{\circ}$. The extensive observations in the latitude range $\pm 10^{\circ}$ recently completed at Hat Creek provide much information on high-velocity gas in these lower latitudes. We find:

(1) There is much intermediate-velocity and high-velocity gas (preponderantly of negative sign) in the galactic plane. It is not in the form of isolated clouds or concentrations; it is connected to the lower-velocity gas in the Galaxy.

(2) There is much greater tendency for intermediate-velocity and high-velocity gas of high intensity to be present in some longitudes than in others. There are 'active' regions in the Galaxy.

(3) High-velocity features are often jet-like in nature. Jet-like here means that a mass of gas occupying a very limited angular extent in the sky (an area one to a few degrees in diameter) will show a large range in velocity extending, perhaps from $-100 \mathrm{~km} \mathrm{sec}^{-1}$ to the velocity of the normal galactic gas.

An example of a jet-like gas structure mentioned in (3) is shown in Figure 14. The strong jet visible particularly at $l^{\mathrm{II}}=196^{\circ} .5$ has the following characteristics:

$\begin{array}{ll}\text { Effective diameter } & \approx 2^{\circ} \\ \text { Estimated distance } & 250 \mathrm{pc} \\ \text { Total mass } & 185 M_{\odot} \\ \text { Total kinetic energy } & 5 \times 10^{48} \mathrm{erg} \\ \text { Column density of } \mathrm{HI} & 4 \times 10^{20} \mathrm{H} \text { atom cm }^{-2} \\ \text { Average number density of } \mathrm{H} & \leqslant 10 \mathrm{H} \text { atom } \mathrm{cm}^{-3} .\end{array}$

For comparison, we may note that the energy expended by an average nova is $10^{45}$ to $10^{46} \mathrm{erg}$, while that expended by an average supernova is perhaps $10^{50}$ to $10^{51} \mathrm{erg}$.

(In the presentation before the Symposium, a computer-produced movie, made from the Hat Creek observations, was used to illustrate galactic active regions and jet-like gas structures.) 


\section{References}

Barrett, A. H., Meeks, M. L., and Weinreb, S.: 1964, Nature 202, 475.

Blaauw, A.: 1952, Bull. Astron. Inst. Netherl. 11, 459.

Bok, B. J., Lawrence, R. S., and Menon, T. K.: 1955, Publ. Astron. Soc. Pacific 67, 108.

Cheung, A. C., Rank, D. M., Townes, C. H., Thornton, D. D., and Welch, W. J.: 1968, Phys. Rev. Lett. 21, 1701.

Cheung, A. C., Rank, D. M., Townes, C. H., Knowles, S. H., and Sullivan, W. T. III: 1969a, Astrophys. J. Lett., 157, L13.

Cheung, A. C., Rank, D. M., Townes, C. H., Thornton, D. D., and Welch, W. J.: 1969b, Nature 221, 626.

Cheung, A. C., Rank, D. M., Townes, C. H., and Welch, W. J.: 1969c, Nature 221, 917.

Clark, B. G.: 1965, Astrophys. J. 142, 1398.

Davies, R. D.: 1956, Monthly Notices Roy. Astron. Soc. 116, 443.

Davies, R. D.: 1958, Rev. Mod. Phys. 30, 931.

Dieter, N. H.: 1969, Publ. Astron. Soc. Pacific 81, 186.

Field, G. B., Goldsmith, D. W., and Habing, H. J.: 1969, Astrophys. J. Lett. 155, L149. See also

Garzoli, S. L. and Varsavaky, C. M.: 1966, Astrophys. J. 145, 79.

Goldsmith, D. W., Habing, H. J., and Field, G. B.: 1969, Astrophys. J. 158, 173.

Goss, W. M.: 1968, Astrophys. J. Suppl. Ser. 15, 131.

Habing, H. J.: 1969a, Bull. Astron. Inst. Netherl. 20, 120.

Habing, H. J.: 1969b, Bull. Astron. Inst. Netherl. 20, 177.

Heeschen, D. S.: 1955, Astrophys. J. 121, 569.

Heiles, C.: 1967a, Astrophys. J. Suppl. Ser. 15, 97.

Heiles. C.: 1967b, Astrophys. J. 148, 299.

Heiles, C.: 1968, Astrophys. J. 151, 919.

Heiles, C.: 1969, Astrophys. J. 156, 493.

Herbig, G.: 1968, Z. Astrophys. 68, 243.

Hobbs, L. M.: 1969a, Astrophys. J. 157, 135.

Hobbs, L. M.: 1969b, Astrophys. J. 157, 165.

Howard, W. E., Wentzel, D. G., and McGee, R. X.: 1963, Astrophys. J. 138, 988.

Kerr, F. J.: 1964, in IAU Symposium No. 20, The Galaxy and the Magellanic Clouds (ed. by F. J. Kerr and A. W. Rodgers), Australian Academy of Sciences, Canberra, Australia, p. 81.

Kerr, F. J.: 1969, Rev. Astron. Astrophys. 7, 39.

Kerr, F. J. and Garzoli, S.: 1968, Astrophys. J. 152, 51.

Knowles, S. H., Mayer, C. H., Cheung, A. C., Rank, D. M., and Townes, C. H.: 1969, Science 163, 1055.

Lin, C. C.: 1967, Ann. Rev. Astron. Astrophys. 5, 453 (other earlier references will be found in this publication).

Mebold, U.: 1969, Beitr. Radioastron. 1, 97 (Max-Planck-Institut für Radioastronomie Bonn).

Meeks, M. L., Carter, J. C., Barrett, A. H., Schwartz, P. R., Waters, J. W., and Brown, W. E. III.: 1969 (in press).

Mészáros, P.: 1968, Astrophys. Space Sci. 2, 510.

Münch, G.: 1965, in Galactic Structure (ed. by A. Blaauw and M. Schmidt), University of Chicago Press, Chicago, p. 203.

Palmer, P., Zuckerman, B., Buhl, D., and Snyder, L. E.: 1969, Astrophys. J. Lett. 156, L147.

Pronik, I. I.: 1963, Izv. Krymsk. Astrofiz. Observ. 30, 118.

Radhakrishnan, V. and Murray, J. D.: 1969, Proc. Astr. Soc. Australia 1, 215.

Riegel, K. W. and Jennings, M. C.: 1969, Astrophys. J. 157, 563.

Roberts, Jr., W. W.: 1969, Astrophys. J. 158, 123.

Roberts, Jr., W. W.: 1970, in IAU Symposium No. 38, The Spiral Structure of Our Galaxy (ed. by W. Becker and G. Contopoulos), Reidel, Dordrecht, The Netherlands, p. 415.

Rydbeck, O. E. H., Ellder, J., and Kollberg, E.: 1969 (in press).

Sandage, A. R.: 1961, The Hubble Atlas of Galaxies, Carnegie Institution of Washington.

Schmidt, M.: 1957, Bull. Astron. Inst. Netherl. 13, 247.

Schmidt, M.: 1965, in Galactic Structure (ed. by A. Blaauw and M. Schmidt), University of Chicago Press, Chicago, p. 513. 
Schmidt-Kaler, T.: 1964, Trans. IAU 12B, 416.

Shuter, W. L. H. and Verschuur, G. L.: 1964, Monthly Notices Roy. Astron. Soc. 127, 387.

Snyder, L. E., Buhl, D., Zuckerman, B., and Palmer, P.: 1969, Phys. Rev. Lett. 22, 679.

Takakubo, K.: 1967, Bull. Astron. Inst. Netherl. 19, 125.

Turner, B. E.: 1969, Astrophys. J. 157, 103. See also Ball, J. A. and Staelin, D. H.: 1968, Astrophys. J. Lett. 153, L41.

Van de Hulst, H. C., Muller, C. A , and Oort, J. H.: 1954, Bull. Astron. Inst. Netherl. 12, 117.

Van Woerden, H.: 1967, in IAU Symposium No. 31, Radio Astronomy and the Galactic System (ed. by H. van Woerden), Academic Press, London, p. 3.

Varsavsky, C. M.: 1968, Astrophys. J. 153, 627.

Weaver, H.: 1970, in IAU Symposium No. 38, The Spiral Structure of Our Galaxy, Basel, Switzerland (ed. by W. Becker and G. Contopoulos), Reidel, Dordrecht, The Netherlands, p. 126.

Weaver, H. and Williams, D. R. W.: 1970 (in preparation).

Weaver, H., Dieter, N. H., and Williams, D. R. W.: 1968, Astrophys. J. Suppl. Ser. 16, 219.

Westerhout, G.: 1957, Bull. Astron. Inst. Netherl. 13, 201.

Williams, D. R. W.: 1969, private communication.

Wilson, W. J. and Barrett, A. H.: 1968, Science 161, 778, 23. 